$10-2-2013$

\title{
On the Finite Sample Properties of Pre-test Estimators of Spatial Models
}

Gianfranco Piras

Gianfranco.Piras@mail.wvu.edu

Ingmar R. Prucha

prucha@econ.umd.edu

Follow this and additional works at: https://researchrepository.wvu.edu/rri_pubs

Part of the Regional Economics Commons

\section{Digital Commons Citation}

Piras, Gianfranco and Prucha, Ingmar R., "On the Finite Sample Properties of Pre-test Estimators of Spatial Models" (2013). Regional Research Institute Publications and Working Papers. 15.

https://researchrepository.wvu.edu/rri_pubs/15 accepted for inclusion in Regional Research Institute Publications and Working Papers by an authorized administrator of The Research Repository @ WVU. For more information, please contact ian.harmon@mail.wvu.edu. 


\title{
On the finite sample properties of pre-test estimators of spatial models
}

\author{
Gianfranco Piras* Ingmar R. Prucha ${ }^{\dagger}$
}

October 2, 2013

\begin{abstract}
This paper explores the properties of pre-test strategies in estimating a linear Cliff-Ord-type spatial model when the researcher is unsure about the nature of the spatial dependence. More specifically, the paper explores the finite sample properties of the pre-test estimators introduced in Florax et al. (2003), which are based on Lagrange Multiplier (LM) tests, within the context of a Monte Carlo study. The performance of those estimators is compared with that of the maximum likelihood (ML) estimator of the encompassing model. We find that, even in a very simple setting, the bias of the estimates generated by pre-testing strategies can be very large in some cases and the empirical size of tests can differ substantially from the nominal size. This is in contrast to the ML estimator.
\end{abstract}

\section{Introduction}

The recent literature on spatial econometrics has been concerned with model specification issues both in cross sectional as well as panel data analysis. ${ }^{1}$ In

${ }^{*}$ Regional Research Institute, West Virginia University, Morgantown, WV 26506, Tel.: +1-304-293-2643, e-mail: Gianfranco.Piras@mail.wvu.edu

${ }^{\dagger}$ Department of Economics, University of Maryland, College Park, MD 20742, Tel.: +1-301-405-3499, e-mail: prucha@econ.umd.edu

${ }^{1}$ See e.g., Cliff \& Ord (1972, 1973, 1981); Florax \& Folmer (1992); Anselin et al. (1996); Anselin (1988a); Florax et al. (2003); Baltagi et al. (2007, 2003); Baltagi \& Liu (2008); Baltagi et al. (2009, 2008); Burridge (1980); Debarsy \& Ertur (2010), among many others. 
the present paper we will focus exclusively on cross sectional models. Empirical work is often based on estimation strategies which entails estimating initially a linear model (i.e., without spatial dependencies), followed by testing for spatial dependences, and re-estimation if spatial dependence cannot be rejected. It is well known that, in general, pre-test strategies may potentially introduce bias for both the parameter estimates and corresponding standard errors; see, e.g., Leeb \& Poetscher (2008). Of course, on the other hand, efficiency may be lost when the researcher estimates a more general model than necessary. The purpose of this paper is to explore the implications of some common pre-test strategies used in the estimation of Cliff-Ord-type spatial models.

In estimating Cliff-Ord-type models two forms of spatial dependences are usually considered in applied work, which correspond to two different model specifications. The first form of spatial dependence relates to the error term and specifies a spatial auto regressive process for the disturbances. Correspondingly, the model that derives from it is often referred to as a spatial error model (see, e.g., Anselin, 1988b). The second form arises when the value of the dependent variable corresponding to each cross-sectional unit is jointly determined with the values at all other neighboring cross-sectional units. This is achieved through the inclusion of a weighted average of the dependent variable which is often described in the literature as a spatial lag. Consequently, the model that derives from it is often referred to as a spatial autoregressive model or, simply, a spatial lag model (see, e.g., Anselin, 1988b).

Burridge (1980) and Anselin et al. (1996) propose simple LM diagnostic tests, based on Ordinary Least Squares (OLS) residuals, for spatial error autocorrelation or spatial lag dependence. More recently Florax et al. (2003) suggest a simple selection criterion conditional upon the results of these specification tests. It should be noted that this testing strategy only leads to the estimation of either the spatial lag or the spatial error models and never of a model that contains both error and lag dependences, i.e., of the encompassing or full model. A partial explanation for this may be that the parameters of the full model are not identified if the model does not contain exogenous variables. However, most empirical specifications include exogenous variables, and in this situation the parameters of the full model are identified under mild regularity conditions; see, e.g., Kelejian \& Prucha (1998). Thus in this situation the researcher can estimate the parameters of the full model, and is not forced to select either the spatial lag or error model. Since the 
data may have been generated by the full model, which includes the spatial lag and error model as special cases, this reduces the likelihood of model misspecification. Of course, if the data have been generated by either the spatial lag or error model, estimating the full model will lead to a loss of efficiency. On the other hand, using a pre-testing strategy may yield biased estimates and may result in a situation where the employed asymptotic distribution of the estimator (derived without taking into account the pre-testing strategy) provides a bad approximation to the actual small sample distribution of the final stage estimator.

This paper explores the importance of the issues raised above for the estimation of linear Cliff-Ord-type spatial models. We explore within the context of a Monte Carlo study the small sample performance of three pretest estimators which are described in Florax et al. (2003) and which are based on a series of LM tests. We also compare their performance with that of the ML estimator of the general model, which allows for spatial spill-overs in the endogenous variables and disturbances. Our results are cautionary, in that we find that even in simple settings the bias of the estimates generated by a pre-testing strategy can be very large and the empirical size of tests can differ substantially from the nominal size. Quite expectedly, the results also show that the ML estimator based on the full model is consistent, and the size of hypothesis tests is reasonably close to the nominal size.

Section 2 briefly describes the models considered for this study and presents the corresponding likelihoods on which our estimators are based. Section 3 introduces the LM tests while Section 4 gives the three pre-test estimators based on those LM tests. Section 5 describes the design of our Monte Carlo experiment and discusses the main evidence. Section 6 concludes and give indication for future work.

\section{The models}

As we mentioned in the introduction, much of the empirical spatial econometrics literature has focused on the estimation of two alternative models relating to different forms of spatial dependence. In one case, spatial dependence is introduced via the disturbance process, where the disturbance term corresponding to one location is assumed to be jointly determined with those at other locations. In the other case, the dependent variable at one location is assumed to be jointly determined by its values at other locations. From an 
empirical perspective, each of these two forms of dependence translates into a different Cliff-Ord-type spatial model. The model corresponding to the first case is known in the literature as spatial error model; while the model corresponding to the second case is known as the spatial lag model (Cliff \& Ord, 1973; Anselin, 1988b). In what follows, we will briefly review these models and the corresponding likelihoods. Towards assessing the effects of pre-test strategies we will furthermore consider the encompassing Cliff-Ord-type spatial model, which includes both forms of spatial effects. As is common in the literature we refer to this model as a spatial auto-regressive auto-regressive model (SARAR(1,1)); see e.g., Anselin (1988b). As remarked, the parameters of the SARAR $(1,1)$ model can be consistently estimated under mild regularity conditions, provided the presence of exogenous variables; see, e.g., Kelejian \& Prucha (1999, 2010); Florax \& Folmer (1992); Anselin (1988b). Of course, if the true data generating process corresponds to a spatial error or lag model, we expect some loss in efficiency when estimating the encompassing SARAR(1,1) model. We will use the encompassing model to explore the properties of the considered pre-test estimators based on LM tests.

The approach frequently taken in empirical work is to start with the classical linear regression model

$$
y=X \beta+\varepsilon,
$$

where $y$ is an $n \times 1$ vector of observations on the dependent variable, $X$ is an $n \times k$ matrix of observations on the non-stochastic explanatory variables, $\beta$ a $k \times 1$ vector of corresponding parameters, and $\varepsilon$ an $n \times 1$ vector of innovations whose elements are - for simplicity - assumed in the following to be i.i.d. $N\left(0, \sigma^{2}\right){ }^{2}$ Under regularity conditions the OLS estimator is also the ML estimator.

As an alternative to the linear regression model (1), the error term can be specified as a spatial autoregressive process, leading to the spatial error model

$$
\begin{aligned}
& y=X \beta+u, \\
& u=\rho W u+\varepsilon,
\end{aligned}
$$

where $u$ is the $n \times 1$ vector of disturbances, $W$ is an $n \times n$ non-stochastic

\footnotetext{
${ }^{2}$ We note that even for this standard setup our Monte Carlo results will (depending on the parameter constellations) detect sizable biases of the considered pre-test estimators.
} 
weighting matrix, ${ }^{3} \rho$ is a scalar spatial autoregressive parameter with $|\rho|<1$, and all other variables are defined as above. For efficiency we can estimate the model in (2) by ML (Ord, 1975), although OLS remains unbiased. The expression for the log likelihood function of model (2) takes the form

$$
L=-\frac{n}{2} \ln (2 \pi)-\frac{n}{2} \ln \left(\sigma^{2}\right)+\ln |B|-\frac{1}{2 \sigma^{2}}(y-X \beta)^{\prime} B^{\prime} B(y-X \beta),
$$

where $B=I_{N}-\rho W{ }^{4}$ As an alternative to the ML estimator, a feasible GLS estimator, which utilizes a generalized method of moments estimator for $\rho$, has been suggested by Kelejian \& Prucha (1999). However, in this paper we concentrate on the ML estimator.

A further alternative to the linear regression model (1) which is often estimated in the empirical literature is the spatial autoregressive model

$$
y=\lambda W y+X \beta+\varepsilon,
$$

where $\lambda$ is a scalar spatial autoregressive parameter with $|\lambda|<1$, and all other variables are defined as above. Because of the simultaneous nature of the spatial lag variable, $W y$ is correlated with the disturbance term $\varepsilon$. Thus OLS is inconsistent, but the model can again be estimated efficiently by ML. The log likelihood takes the following form (Ord, 1975)

$$
L=-\frac{n}{2} \ln (2 \pi)-\frac{n}{2} \ln \left(\sigma^{2}\right)+\ln |A|-\frac{1}{2 \sigma^{2}}(A y-X \beta)^{\prime}(A y-X \beta),
$$

where $A=I-\lambda W$. As an alternative to the ML estimator, the model could also be estimated by instrumental variables/generalized method of moments (Kelejian \& Prucha, 1998), but again the present paper will focus on the ML estimator.

Finally, we consider the encompassing model which allows for spatial lags in the dependent variable, as well as in the disturbances, i.e.,

$$
\begin{aligned}
& y=\lambda W y+X \beta+u, \\
& u=\rho W u+\varepsilon .
\end{aligned}
$$

\footnotetext{
${ }^{3}$ The assumptions made on the weights matrix are standard and we will not discuss them in this paper.

${ }^{4}$ For details on the maximum likelihood estimation see Anselin (1988b), Ch 12.
} 
The log likelihood for this model is given by

$$
\begin{aligned}
L= & -\frac{n}{2} \ln (2 \pi)-\frac{n}{2} \ln \left(\sigma^{2}\right)+\frac{n}{2} \ln |B|+\frac{n}{2} \ln |A| \\
& -\frac{1}{2 \sigma^{2}}(A y-X \beta)^{\prime} B^{\prime} B(A y-X \beta) .
\end{aligned}
$$

\section{$3 \quad$ LM tests}

In this section we define the LM tests for the absence of spatial dependence employed in the construction of our considered pretest estimators. Burridge (1980) derived the LM test statistic for the spatial error model. Anselin (1988b) derived LM tests for the more general SARAR model. Requiring only the estimation of the restricted specification, LM tests have been considered particularly appealing in a spatial setting because of the computational difficulties related to the maximum likelihood estimation of the spatial models. $^{5}$

With the first of these tests we wish to evaluate the hypothesis that the disturbances are independently normally distributed with constant variance (i.e. $\rho=0$ ) against the alternative that they are generated by the first order spatial autoregression in (2). The LM-test statistics for this hypothesis is given by

$$
L M_{\rho}=\frac{\left[e^{\prime} W e /\left(e^{\prime} e / n\right)\right]^{2}}{\operatorname{tr}\left[W^{\prime} W+W W\right]}
$$

where $e=y-X \widehat{\beta}_{O L S}$ denotes the vector of OLS residuals, and $t r$ is the trace operator.

The second LM-test statistics, which evaluates the null hypothesis that $\lambda=0$ in (4) against the alternative of a spatial autoregressive process is given by

$$
L M_{\lambda}=\left[e^{\prime} W y /\left(e^{\prime} e / n\right)\right]^{2} / D
$$

where $e=y-X \widehat{\beta}_{O L S}$ denotes, as before, the vector of OLS residuals, $D=$ $\left[\left(W X \widehat{\beta}_{O L S}\right)^{\prime} M\left(W X \widehat{\beta}_{O L S}\right) / \widehat{\sigma}_{O L S}^{2}\right]+\operatorname{tr}\left(W^{\prime} W+W W\right), M=\left[I-X\left(X^{\prime} X\right)^{-1} X^{\prime}\right]$, and $\widehat{\beta}_{O L S}$ and $\widehat{\sigma}_{O L S}^{2}$ are the OLS estimates of $\beta$ and $\sigma^{2}$. Under the null hypothesis that the true model is (1) both $L M_{\rho}$ and $L M_{\lambda}$ are asymptotically distributed as $\chi^{2}(1)$.

\footnotetext{
${ }^{5}$ However, with the increase in power achieved by the modern computers, and the various methods to approximate the Jacobian term (LeSage \& Pace, 2009), this problem has been somewhat mitigated.
} 
The two test statistics presented above assume that the other form of dependence is not present. In other word, $L M_{\rho}$ is derived under the null hypothesis that $\rho=0$, but it assumes that also $\lambda$ is zero. Using the general principles of specification testing with locally misspecified alternatives derived in Bera \& Yoon (1993), Anselin et al. (1996) develop a set of diagnostics that are a robust version of (8) and (9). The expressions for the robust versions of the tests become, respectively,

$$
L M_{\rho}^{*}=\frac{\left\{e^{\prime} W e /\left(e^{\prime} e / n\right)-\left[\operatorname{tr}\left(W W+W^{\prime} W\right) / D\right] e^{\prime} W y /\left(e^{\prime} e / n\right)\right\}^{2}}{\operatorname{tr}\left(W W+W^{\prime} W\right) /\left[1-\operatorname{tr}\left(W W+W^{\prime} W\right) / D\right]}
$$

and

$$
L M_{\lambda}^{*}=\frac{\left[e^{\prime} W y /\left(e^{\prime} e / n\right)-e^{\prime} W e /\left(e^{\prime} e / n\right)\right]^{2}}{\left[D-\operatorname{tr}\left(W W+W^{\prime} W\right)\right]}
$$

where $D$ as well as the other symbols where defined before. Both the $L M_{\rho}^{*}$ and the $L M_{\lambda}^{*}$ statistics are asymptotically distributed as $\chi^{2}(1)$.

\section{Pre-test estimators}

The pre-tests estimators are based on the sequence of the LM-tests presented in the previous section. We follow the algorithms illustrated in Florax et al. (2003), which proposes three different "approaches" toward specification tests, each leading to a different pre-test estimator. Before reviewing these three approaches, we need to introduce some additional notation.

Let $\widehat{\beta}_{O L S}$ be the OLS estimator based on model (1), $\widehat{\rho}_{M L E}$ and $\widehat{\beta}_{M L E}$ the ML estimators corresponding to the model in (2) and, finally, $\widehat{\lambda}_{M L L}$ and $\widehat{\beta}_{M L L}$ the ML estimators corresponding to the model in (4). Also, let $\widehat{\lambda}_{M L}$, $\widehat{\rho}_{M L}$, and $\widehat{\beta}_{M L}$ denote the ML estimator for $\lambda, \rho$ and $\beta$ based on the full model in (6). Correspondingly, we have the following estimators for $\theta=\left(\lambda, \rho, \beta^{\prime}\right)^{\prime}$ :

$$
\begin{aligned}
\widehat{\theta}_{O L S} & =\left(0,0, \widehat{\beta}_{O L S}^{\prime}\right)^{\prime}, \\
\widehat{\theta}_{M L E} & =\left(0, \widehat{\rho}_{M L E}, \widehat{\beta}_{M L E}^{\prime}\right)^{\prime}, \\
\widehat{\theta}_{M L L} & =\left(\widehat{\lambda}_{M L L}, 0, \widehat{\beta}_{M L L}^{\prime}\right)^{\prime}, \\
\widehat{\theta}_{M L} & =\left(\widehat{\lambda}_{M L}, \widehat{\rho}_{M L}, \widehat{\beta}_{M L}^{\prime}\right)^{\prime} .
\end{aligned}
$$

The first approach in Florax et al. (2003) is based on the test statistics $L M_{\rho}$ and $L M_{\lambda}$ and can be summarized as follows: 
1. Estimate the non-spatial model by OLS to obtain $\widehat{\beta}_{O L S}$ and the vector of OLS residuals $e=y-X \widehat{\beta}_{O L S}$.

2. Test the hypothesis of no spatial dependence due to an omitted spatially autoregressive error or to an omitted spatial lag using, respectively, $L M_{\rho}$ and $L M_{\lambda}$.

3. If both tests statistics are not significant, then accept $H_{0}: \lambda=\rho=0$, and consequently the estimator for $\theta$ is given by $\widehat{\theta}_{O L S}=\left(0,0, \widehat{\beta}_{O L S}^{\prime}\right)^{\prime}$.

4. If $L M_{\rho}$ is significant and $L M_{\lambda}$ is not significant then accept $H_{1}^{\rho}: \lambda=$ $0 ; \rho \neq 0$ and estimate model (2) by maximum likelihood to get $\widehat{\theta}_{M L E}=$ $\left(0, \widehat{\rho}_{M L E}, \widehat{\beta}_{M L E}^{\prime}\right)^{\prime}$.

5. If $L M_{\lambda}$ is significant and $L M_{\rho}$ is not significant then accept $H_{1}^{\lambda}: \lambda \neq$ $0 ; \rho=0$ and estimate model (4) by maximum likelihood to get $\widehat{\theta}_{M L L}=$ $\left(\widehat{\lambda}_{M L L}, 0, \widehat{\beta}_{M L L}^{\prime}\right)^{\prime}$.

6. Finally, if both $L M_{\lambda}$ and $L M_{\rho}$ are significant, estimate the specification corresponding to the more significant of the two tests.

Florax et al. (2003) refer to this approach as the "classic" approach because it is based on the test statistics $L M_{\rho}$ and $L M_{\lambda}$.

Let $\widehat{\theta}_{P T 1}$ denote the estimator for $\theta$ based on this approach, where "PT" stands for "pre-test". Then this estimator is formally given by

$$
\begin{aligned}
\widehat{\theta}_{P T 1}= & \left(\widehat{\lambda}_{P T 1}, \widehat{\rho}_{P T 1}, \widehat{\beta}_{P T 1}^{\prime}\right)^{\prime} \\
= & \mathbf{1}\left(L M_{\lambda}<\chi_{.95}, L M_{\rho}<\chi_{.95}\right) \widehat{\theta}_{O L S} \\
& +\mathbf{1}\left(L M_{\lambda}<\chi_{.95}, L M_{\rho} \geq \chi_{.95}\right) \widehat{\theta}_{M L E} \\
& +\mathbf{1}\left(L M_{\lambda} \geq \chi_{.95}, L M_{\rho}<\chi_{.95}\right) \widehat{\theta}_{M L L} \\
& +\mathbf{1}\left(L M_{\lambda} \geq \chi_{.95}, L M_{\rho} \geq \chi_{.95}\right) \mathbf{1}\left(L M_{\lambda}<L M_{\rho}\right) \widehat{\theta}_{M L E} \\
& +\mathbf{1}\left(L M_{\lambda} \geq \chi_{.95}, L M_{\rho} \geq \chi_{.95}\right) \mathbf{1}\left(L M_{\lambda} \geq L M_{\rho}\right) \widehat{\theta}_{M L L} .
\end{aligned}
$$

where $\mathbf{1}($.$) denotes the indicator function.$

The second approach, that they refer to as the robust approach, is identical to the previous one with the exception that it is performed with the 
robust versions of the LM tests. Let $\widehat{\theta}_{P T 2}$ denote the estimator for $\theta$ based on this approach, then it is formally given by

$$
\begin{aligned}
\widehat{\theta}_{P T 2}= & \left(\widehat{\lambda}_{P T 2}, \widehat{\rho}_{P T 2}, \widehat{\beta}_{P T 2}^{\prime}\right)^{\prime} \\
= & \mathbf{1}\left(L M_{\lambda}^{*}<\chi_{.95}, L M_{\rho}^{*}<\chi_{.95}\right) \widehat{\theta}_{O L S} \\
& +\mathbf{1}\left(L M_{\lambda}^{*}<\chi_{.95}, L M_{\rho}^{*} \geq \chi_{.95}\right) \widehat{\theta}_{M L E} \\
& +\mathbf{1}\left(L M_{\lambda}^{*} \geq \chi_{.95}, L M_{\rho}^{*}<\chi_{.95}\right) \widehat{\theta}_{M L L} \\
& +\mathbf{1}\left(L M_{\lambda}^{*} \geq \chi_{.95}, L M_{\rho}^{*} \geq \chi_{.95}\right) \mathbf{1}\left(L M_{\lambda}^{*}<L M_{\rho}^{*}\right) \widehat{\theta}_{M L E} \\
& +\mathbf{1}\left(L M_{\lambda}^{*} \geq \chi_{.95}, L M_{\rho}^{*} \geq \chi_{.95}\right) \mathbf{1}\left(L M_{\lambda}^{*} \geq L M_{\rho}^{*}\right) \widehat{\theta}_{M L L} .
\end{aligned}
$$

Florax et al. (2003) consider a third approach which is a "hybrid" specification strategy in that it combines the use of both test statistics (classical and robust). It is identical to the classical approach, except that step 6 is modified as follows: If both $L M_{\rho}$ and $L M_{\lambda}$ are significant, estimate the specification pointed by the more significant of the two robust statistics $L M_{\rho}^{*}$ and $L M_{\lambda}^{*}$. As pointed out by Florax et al. (2003) the performance of this hybrid pre-test estimator is identical to the classical pre-test estimator. The reason is that, analytically, $L M_{\lambda} \geq L M_{\rho}$ if and only if $L M_{\lambda}^{*} \geq L M_{\rho}^{*}$, as is easily checked. ${ }^{6}$ We thus do not report separately on the performance of this hybrid pre-test estimator.

From the above definitions of the pre-test estimators it is obvious that they are highly nonlinear, and that they cannot generally be expected to be unbiased or consistent. Furthermore, it is obvious that the asymptotic distribution of those estimators will generally differ from those of $\widehat{\theta}_{O L S}, \widehat{\theta}_{M L L}$ and $\widehat{\theta}_{M L E}$. Of course, potential issues stemming from improper inference when using pre-test estimators are well known; see, e.g., Leeb \& Poetscher (2008). The Monte Carlo study is intended to shed some light on the importance of those issues in the estimation of spatial models.

\section{Monte Carlo}

In what follows, we report on a Monte Carlo study of the small sample properties of the two pre-test estimators defined above. For comparison we also give results for the small sample properties of the ML estimator

\footnotetext{
${ }^{6}$ Results on this are available from the authors.
} 
defined by the log-likelihood function of the comprehensive model (6). The design of the Monte Carlo is, intentionally, very simple. Our findings suggest that even for simple settings the bias of the estimates generated by pretesting strategies can be very large and the empirical size of tests can differ substantially from the nominal size. This is in contrast to the ML estimator.

\section{Monte Carlo Design}

In all of the experiments, the data are generated from the following simple model:

$$
\begin{aligned}
& y=\lambda W y+x_{1} \beta_{1}+x_{2} \beta_{2}+u, \\
& u=\rho W u+\varepsilon .
\end{aligned}
$$

Obviously, for $\lambda=0$ or $\rho=0$ this model includes, respectively, the spatial error or spatial lag model as special cases. The two regressors, $x_{1}$ and $x_{2}$, are normalized versions of income per capita and the proportion of housing units that are rental in 1980, in 760 counties in U.S. mid-western states. ${ }^{7}$ We normalized the data by subtracting from each observation the corresponding sample average, and then dividing that result by the sample standard deviation. The first $n$ values of these normalized variables were used in our Monte Carlo experiments. The regressors are treated as fixed and thus are held constant over all of the Monte Carlo trials. The Monte Carlo study assumes that units are located on a regular grid of dimension $23 \times 23$, which implies a sample size of $n=529$. The spatial weighting matrix employed in our Monte Carlo study is based on the queen criteria (i.e. common borders and vertex). The values of $\beta_{1}$ and $\beta_{2}$ are set equal to one. We consider the same set of values for both $\rho$ and $\lambda$, namely $-0.8,-0.6,-0.4,-0.2,0,0.2,0.4$, $0.6,0.8$. Finally, we assume that the elements of the innovation vector are i.i.d. $N(0,1){ }^{8}$ The results presented in the next section are based on 1,000 replications. All elaborations were performed using $\mathrm{R}$ statistical software ( $\mathrm{R}$ Development Core Team, 2010) with the library spdep (Bivand et al. , 2010). ${ }^{9}$

\footnotetext{
${ }^{7}$ These data were taken from Kelejian \& Robinson (1995) and where also used by Arraiz et al. (2010).

${ }^{8}$ The target $R^{2}$ for the simulation was set to 0.6 .

${ }^{9}$ We also run a Monte Carlo simulations with weighting matrix based on the rook criteria, and another simulations with a larger sample size (i.e., $n=1,024$ ). The results
} 


\section{Monte Carlo Results}

The results of the Monte Carlo experiments are reported in Tables 1-6. The first four tables relate to the bias and mean squared errors (MSEs) of the two pretest estimators and the ML estimator of, respectively, $\rho, \lambda, \beta_{1}$, and $\beta_{2}$. In all tables, the first two columns contain the considered combinations of the true values of $\rho$ and $\lambda$ employed in generating the data. We note again that if $\lambda=0$ the data are generated from a spatial error model, and if $\rho=0$ the data are generated from a spatial lag model. In columns three to five we report the biases of the respective estimators, and in columns six to eight the MSEs.

For additional insight we also report in Table 5 the frequency with which the two considered pre-test procedures select the classical linear regression model (1), the spatial error model (2) and spatial lag model (4). Finally, in Table 6 we report on the empirical size of tests of the hypothesis that respectively $\beta_{1}$ and $\beta_{2}$ are equal to the true parameter value. More specifically, all tests are Wald tests based on "t-ratios", where the standard errors are calculated from the negative inverse Hessian of the log-likelihood function. For the pre-test estimators - consistent with what seems to be the usual practice when such estimators are employed in empirical work - these calculations are based on the log-likelihood function corresponding to the model selected by the pre-test procedure. For the ML estimator the calculations are based

on the log-likelihood function of the full model. We next discuss the results in Tables 1-6 in more detail.

Consider first the results in Table 1. Looking at the averages, we note that the bias of $\widehat{\rho}_{M L}$ is considerably lower (0.0079) than that of the pre-test estimators $\widehat{\rho}_{P T 1}$ and $\widehat{\rho}_{P T 2}(0.2583$ and 0.2587$)$. The highest bias corresponds to situations where there is substantial spatial dependence both of the error and of the lag type. As an example, consider the first row when both $\rho$ and $\lambda$ are equal to -0.8 . The bias for both pre-test estimators in this case equals 0.8. This is because both pre-test procedures always lead to the estimation of the spatial lag model for this combination of values; see the first line of Table 5. The bias is still very high when $\rho=-0.8$ and $\lambda$ equals to -0.6 or -0.4 because even in these cases the pre-test procedure tend to suggest overwhelmingly the estimation of a spatial lag model. Interestingly, when

are qualitatively similar to the ones reported here. They are available from the authors upon request. We note that the pre-test estimators and associated confidence remain biased even for larger sample sizes. 
moving to positive values of $\rho$ the situation is quite different. For example, when $\rho=\lambda=0.8$, there is still a predominance of selecting the spatial lag model but the frequency of selecting the spatial error model increases to about $33 \%$, and for $\rho=0.8$ and $\lambda=0.6$ the frequency of selecting the spatial error model is close to $90 \%$. In general, apart from very large values of $\rho$ the two pre-test procedures seem to point to the estimation of the spatial lag model. The tables also show that when one of the spatial parameters is zero the difference in terms of bias declines considerably.

In looking at the MSEs reported in Table 1 we see that the MSEs of the two pretest estimators for $\rho$ are very similar, while (on average) the MSE of the ML estimator is considerably lower (0.1417 vs. 0.0087). Not surprisingly, when either $\lambda=0$ or $\rho=0$ (i.e., there is only one form of spatial dependence), the MSE of $\widehat{\rho}_{M L}$ is generally higher than that of the pre-test estimators.

Table 2 displays the bias and MSE for the estimators of $\lambda$. Consider first the results in terms of bias. Looking at the averages, the results for the estimators of $\lambda$ are in line with what was found for the estimators of $\rho$. More specifically, the average bias for $\widehat{\lambda}_{M L}$ is considerably lower (0.0029) than those of the pre-test estimators $\widehat{\lambda}_{P T 1}$ and $\widehat{\lambda}_{P T 2}(0.1328$ and 0.1324$)$. There are situations, however, in which the pre-test estimators perform very well. Not surprisingly these situations relate to cases where at least one of the spatial parameters is zero. For example, when $\rho=-0.8$ and $\lambda=0$ both pre-test procedures always suggest the estimation of the error model - see Table 5 , line $5-$, and thus the bias of both $\widehat{\lambda}_{P T 1}$ and $\widehat{\lambda}_{P T 2}$ is equal to zero. Results for the MSE are also in line with the findings for the estimators for $\rho$, with the MSE of $\widehat{\lambda}_{M L}$ being substantially lower (0.0038) to those of either $\widehat{\lambda}_{P T 1}$ and $\widehat{\lambda}_{P T 2}$ (0.0519 and 0.0518). Again not surprisingly, when either $\lambda=0$ or $\rho=0$, the MSE of $\widehat{\lambda}_{M L}$ is generally higher than the MSE of the pre-test estimators.

The results in Tables 3 and 4 pertain to the bias and MSE of the estimates of $\beta_{1}$ and $\beta_{2}$, respectively. Since the results for the two parameters are very similar, we will just focus on the results for $\beta_{1}$. On average, the bias of both $\widehat{\beta}_{1, P T 1}$ and $\widehat{\beta}_{1, P T 2}$ is larger than that of $\widehat{\beta}_{1, M L}$. For some parameter constellations the bias of the pretest procedures can be sizable. In particular, for $\rho=0.8$ and $\lambda=-0.8$ the bias of $\widehat{\beta}_{1, P T 1}$ and $\widehat{\beta}_{1, P T 2}$ is 0.1401 , and that of $\widehat{\beta}_{1, M L}$ is 0.0016 . However, when $\rho$ is equal zero (e.g., there is no error dependence), both pre-test estimators perform very similarly to the $\widehat{\beta}_{1, M L}$. Additionally, when the data generating model is the simple classical linear 
regression model (i.e., both $\rho$ and $\lambda$ are zero), both pre-test estimators, $\widehat{\beta}_{1, P T 1}$ and $\widehat{\beta}_{1, P T 2}$, have a smaller bias $(-0.0017$ and -0.0014$)$ than $\widehat{\beta}_{1, M L}(-0.0031)$. Of course, when there is substantial spatial dependence (both coefficients are different from zero), $\widehat{\beta}_{1, M L}$ always outperforms the two pre-test estimators.

The results reported in Tables 1-4 suggest that, even for very simple spatial data generating processes as those underlying our Monte Carlo analysis, the bias of the pre-test estimators can be very large in many cases. On the other hand, the ML estimators based on the full model are consistent for all the parameter values.

We next discuss the results reported in Table 6. As discussed, this table display the results on the empirical size of a Wald test of the hypothesis that a respective parameter is equal to the true parameter value, where the intended size is $5 \%$. Again we will focus our discussion on test pertaining to $\beta_{1}$. For the pretest estimators on average the size of the test is, respectively, $16 \%$ and $12 \%$. However, in some experiments it can be as large as $80 \%$. In particular, it reaches the value of $82 \%$ when $\rho=-0.8$ and $\lambda=-0.2$. For the ML estimator the average empirical size of the test is $5.2 \%$ and thus close to the nominal size of $5 \%$. For the different parameter constellations considered the size of the test remains close to the nominal size and varies between $3.7 \%$ and $6 \%$.

\section{Conclusions}

This paper examined the small sample properties of model selection and pretest procedures in spatial econometrics. In particular, we consider a crosssectional Cliff-Ord-type model, and examine the small sample properties of pre-test estimators suggested in Florax et al. (2003). We also explore the properties of corresponding Wald tests. For comparison we also report on the small sample properties of the ML estimator for the comprehensive model, and corresponding Wald tests.

Our Monte Carlo design is purposefully kept simple to avoid the contamination of the results from other modeling issues. For the same reason we draw the innovations from a Gaussian distribution, and we use the ML approach based on a Gaussian likelihood in the estimation of sub models and of the comprehensive model. The tests employed in the pre-test are likelihood ratio tests based on a Gaussian likelihood. Even within our simple setup we find that the biases of the estimators generated by the pre-testing strategies 
can be large and the size of hypothesis test may be quite different from the envisioned nominal size. In contrast, and not surprising, the ML estimator based on the comprehensive model is consistent, and the size of hypothesis tests is reasonably close to the nominal values. We also find that the loss in efficiency of estimating the full model, when the data are actually generated by a sub model, is modest.

We understand that most empirical work involves a certain amount of model selection and pretesting. However, our results suggest that for the estimation of a cross-sectional Cliff-Ord-type model pre-test strategies may be associated with substantial pitfalls. Our results also suggest that empirical researchers may seriously entertains estimating the comprehensive model, given that the potential efficiency losses from estimating the comprehensive model (when $\rho$ or $\lambda$ are zero) can be modest.

Recently, Debarsy \& Ertur (2010) suggested an extension of the pre-test estimators considered by Florax et al. (2003) to a panel framework. In future research it may be of interest to explore the properties of those extensions to a panel data setting.

\section{References}

Anselin, L. 1988a. Lagrange multiplier test diagnostics for spatial dependence and spatial heterogeneity. Geographical Analysis, 20, 1-17.

Anselin, L. 1988b. Spatial Econometrics: Methods and Models. Dordrecht: Kluwer Academic Publisher.

Anselin, L., Bera, A.K., Florax, R.J.G.M., \& Yoon, M.J. 1996. Simple diagnostic tests for spatial dependence. Regional Science and Urban Economics, 26, 77-104.

Arraiz, Irani, Drukker, David M., Kelejian, Harry H., \& Prucha, Ingmar R. 2010. A spatial Cliff-Ord-type model with heteroskedastic innovations: small and large sample results. Journal of Regional Science, 50(2), 592614 .

Baltagi, B.H., \& Liu, L. 2008. Testing for random effects and spatial lag dependence in panel data models. Statistics and Probability Letters, 78, 3304-3306. 
Baltagi, B.H., Song, S.H., \& Koh, W. 2003. Testing panel data regression models with spatial error correlation. Journal of Econometrics, 117, 123150.

Baltagi, B.H., Song, S.H., Jung, B.C., \& Koh, W. 2007. Testing for serial correlation, spatial autocorrelation and random effects using panel data. Journal of Econometrics, 140(1), 5-51.

Baltagi, B.H., Egger, P., \& Pfaffermayr, M. 2008. A Monte Carlo Study for Pure and Pretest Estimators of a Panel Data Model with Spatially Autocorrelated Disturbances. Annales d'Économie et de Statistique, 87/88, $11-38$.

Baltagi, B.H., Egger, P., \& Pfaffermayr, M. 2009. A Generalized Spatial Panel Data Model with Random Effects. CPR-Working paper series.

Bera, A.K., \& Yoon, M.J. 1993. Specification testing with misspecified alternatives. Econometric Theory, 9, 649-658.

Bivand, Roger, with contributions by Micah Altman, Anselin, Luc, Assuncao, Renato, Berke, Olaf, Bernat, Andrew, Blanchet, Guillaume, Blankmeyer, Eric, Carvalho, Marilia, Christensen, Bjarke, Chun, Yongwan, Dormann, Carsten, Dray, Stphane, Halbersma, Rein, Krainski, Elias, Lewin-Koh, Nicholas, Li, Hongfei, Ma, Jielai, Millo, Giovanni, Mueller, Werner, Ono, Hisaji, Peres-Neto, Pedro, Piras, Gianfranco, Reder, Markus, Tiefelsdorf, Michael, , \& Yu., Danlin. 2010. spdep: Spatial dependence: weighting schemes, statistics and models. R package version 0.5-27.

Burridge, P. 1980. On the Cliff-Ord test for spatial correlation. Journal of the Royal Statistical Society B, 42, 107-108.

Cliff, A., \& Ord, J.K. 1972. Testing for Spatial Autocorrelation among Regression Residuals. Geographical Analysis, 4, 267-284.

Cliff, A., \& Ord, J.K. 1973. Spatial Autocorrelation. London: Pion.

Cliff, A., \& Ord, J.K. 1981. Spatial Processes: Models and Applications. London: Pion.

Debarsy, N., \& Ertur, C. 2010. Testing for spatial autocorrelation in a fixed effects panel data model. Regional Science and Urban Economics, 40, 453-470. 
Florax, R.J.G.M., \& Folmer, H. 1992. Specification and estimation of spatial linear regression models: Monte Carlo evaluation of pre-test estimators. Regional Science and Urban Economics, 22, 405-432.

Florax, R.J.G.M., Folmer, H., \& Rey, S.J. 2003. Specification searches in spatial econometrics: the relevance of Hendry's methodology. Regional Science and Urban Economics, 33, 557-579.

Kelejian, H.H., \& Prucha, I.R. 1998. A Generalized Spatial Two Stages Least Square Procedure for Estimating a Spatial Autoregressive Model with Autoregressive Disturbances. Journal of Real Estate Finance and Economics, 17(1), 99-121.

Kelejian, H.H., \& Prucha, I.R. 1999. A Generalized Moments Estimator for the Autoregressive Parameter in a Spatial Model. International Economic Review, 40(2), 509-533.

Kelejian, H.H., \& Prucha, I.R. 2010. Specification and Estimation of Spatial Autoregressive Models with Autoregressive and Heteroscedastic Disturbances. Journal of Econometrics, 157, 53-67.

Kelejian, H.H., \& Robinson, D. 1995. Spatial Correlation: A Suggested Alternative to the Autoregressive Model. Pages 75 - 95 of: Anselin, L., \& Florax, R.J.G.M (eds), New Directions in Spatial Econometrics. New York: Springer.

Leeb, H., \& Poetscher, B.M. 2008. Recent Developments in Model Selection and Related Areas. Econometric Theory, 24, 319-322.

LeSage, J., \& Pace, K.R. 2009. Introduction to Spatial Econometrics. Boca Raton, FL: CRC Press.

Ord, J.K. 1975. Estimation Methods for Models of Spatial Interaction. Journal of American Statistical Association, 70, 120-126.

R Development Core Team. 2010. R: A Language and Environment for Statistical Computing. R Foundation for Statistical Computing, Vienna, Austria. ISBN 3-900051-07-0. 
Table 1: Bias and MSE of $\widehat{\rho}_{P T 1}, \widehat{\rho}_{P T 2}$, and $\widehat{\rho}_{M L}$.

\begin{tabular}{|c|c|c|c|c|c|c|c|}
\hline \multirow[b]{2}{*}{$\rho$} & \multirow[b]{2}{*}{$\lambda$} & \multicolumn{3}{|c|}{ BIAS } & & MSE & \\
\hline & & $\widehat{\rho}_{P T 1}$ & $\widehat{\rho}_{P T 2}$ & $\widehat{\rho}_{M L}$ & $\widehat{\rho}_{P T 1}$ & $\widehat{\rho}_{P T 2}$ & $\widehat{\rho}_{M L}$ \\
\hline-0.8 & -0.8 & 0.8000 & 0.8000 & -0.0002 & 0.6400 & 0.6400 & 0.0125 \\
\hline-0.8 & -0.6 & 0.7946 & 0.7946 & -0.0064 & 0.6387 & 0.6387 & 0.0125 \\
\hline-0.8 & -0.4 & 0.6349 & 0.6349 & -0.0024 & 0.5673 & 0.5673 & 0.0109 \\
\hline-0.8 & -0.2 & -0.0486 & -0.0486 & -0.0034 & 0.1281 & 0.1281 & 0.0114 \\
\hline-0.8 & 0 & -0.0035 & -0.0035 & -0.0006 & 0.0079 & 0.0079 & 0.0102 \\
\hline-0.8 & 0.2 & 0.2608 & 0.2606 & -0.0022 & 0.0781 & 0.0779 & 0.0093 \\
\hline-0.8 & 0.4 & 0.7996 & 0.7996 & -0.0067 & 0.6395 & 0.6395 & 0.0095 \\
\hline-0.8 & 0.6 & 0.8000 & 0.8000 & -0.0068 & 0.6400 & 0.6400 & 0.0084 \\
\hline-0.8 & 0.8 & 0.8000 & 0.8000 & -0.0026 & 0.6400 & 0.6400 & 0.0082 \\
\hline-0.6 & -0.8 & 0.6000 & 0.6000 & -0.0089 & 0.3600 & 0.3600 & 0.0129 \\
\hline-0.6 & -0.6 & 0.6000 & 0.6000 & -0.0044 & 0.3600 & 0.3600 & 0.0120 \\
\hline-0.6 & -0.4 & 0.5627 & 0.5627 & -0.0022 & 0.3531 & 0.3531 & 0.0114 \\
\hline-0.6 & -0.2 & 0.0787 & 0.0787 & -0.0036 & 0.1606 & 0.1606 & 0.0114 \\
\hline-0.6 & 0 & -0.0050 & -0.0050 & -0.0070 & 0.0088 & 0.0088 & 0.0113 \\
\hline-0.6 & 0.2 & 0.3021 & 0.2997 & -0.0037 & 0.1133 & 0.1108 & 0.0098 \\
\hline-0.6 & 0.4 & 0.6000 & 0.6000 & -0.0077 & 0.3600 & 0.3600 & 0.0100 \\
\hline-0.6 & 0.6 & 0.6000 & 0.6000 & -0.0057 & 0.3600 & 0.3600 & 0.0095 \\
\hline-0.6 & 0.8 & 0.6000 & 0.6000 & -0.0030 & 0.3600 & 0.3600 & 0.0099 \\
\hline-0.4 & -0.8 & 0.4000 & 0.4000 & -0.0128 & 0.1600 & 0.1600 & 0.0130 \\
\hline-0.4 & -0.6 & 0.4000 & 0.4000 & -0.0144 & 0.1600 & 0.1600 & 0.0133 \\
\hline-0.4 & -0.4 & 0.3921 & 0.3921 & -0.0081 & 0.1606 & 0.1606 & 0.0121 \\
\hline-0.4 & -0.2 & 0.1581 & 0.1581 & -0.0104 & 0.1246 & 0.1246 & 0.0122 \\
\hline-0.4 & 0 & -0.0011 & 0.0085 & -0.0130 & 0.0123 & 0.0172 & 0.0116 \\
\hline-0.4 & 0.2 & 0.3438 & 0.3328 & -0.0082 & 0.1301 & 0.1231 & 0.0109 \\
\hline-0.4 & 0.4 & 0.4000 & 0.4000 & -0.0084 & 0.1600 & 0.1600 & 0.0097 \\
\hline-0.4 & 0.6 & 0.4000 & 0.4000 & -0.0090 & 0.1600 & 0.1600 & 0.0100 \\
\hline-0.4 & 0.8 & 0.4000 & 0.4000 & -0.0097 & 0.1600 & 0.1600 & 0.0093 \\
\hline-0.2 & -0.8 & 0.2000 & 0.2000 & -0.0104 & 0.0400 & 0.0400 & 0.0123 \\
\hline-0.2 & -0.6 & 0.2000 & 0.2000 & -0.0149 & 0.0400 & 0.0400 & 0.0127 \\
\hline-0.2 & -0.4 & 0.2000 & 0.2000 & -0.0105 & 0.0400 & 0.0400 & 0.0125 \\
\hline-0.2 & -0.2 & 0.1435 & 0.1479 & -0.0082 & 0.0450 & 0.0452 & 0.0110 \\
\hline-0.2 & 0 & 0.0586 & 0.0880 & -0.0049 & 0.0222 & 0.0269 & 0.0109 \\
\hline-0.2 & 0.2 & 0.1994 & 0.1982 & -0.0066 & 0.0399 & 0.0396 & 0.0109 \\
\hline-0.2 & 0.4 & 0.2000 & 0.2000 & -0.0068 & 0.0400 & 0.0400 & 0.0103 \\
\hline-0.2 & 0.6 & 0.2000 & 0.2000 & -0.0067 & 0.0400 & 0.0400 & 0.0091 \\
\hline-0.2 & 0.8 & 0.2000 & 0.2000 & -0.0027 & 0.0400 & 0.0400 & 0.0088 \\
\hline 0 & -0.8 & 0.0000 & 0.0000 & -0.0127 & 0.0000 & 0.0000 & 0.0101 \\
\hline 0 & -0.6 & 0.0000 & 0.0000 & -0.0085 & 0.0000 & 0.0000 & 0.0111 \\
\hline 0 & -0.4 & 0.0000 & 0.0000 & -0.0094 & 0.0000 & 0.0000 & 0.0111 \\
\hline 0 & -0.2 & -0.0075 & -0.0031 & -0.0125 & 0.0024 & 0.0012 & 0.0112 \\
\hline 0 & 0 & -0.0020 & -0.0013 & -0.0143 & 0.0019 & 0.0016 & 0.0114 \\
\hline 0 & 0.2 & 0.0077 & 0.0070 & -0.0078 & 0.0023 & 0.0022 & 0.0094 \\
\hline 0 & 0.4 & 0.0000 & 0.0000 & -0.0037 & 0.0000 & 0.0000 & 0.0110 \\
\hline 0 & 0.6 & 0.0000 & 0.0000 & -0.0053 & 0.0000 & 0.0000 & 0.0092 \\
\hline 0 & 0.8 & 0.0000 & 0.0000 & -0.0071 & 0.0000 & 0.0000 & 0.0082 \\
\hline 0.2 & -0.8 & -0.2000 & -0.2000 & -0.0157 & 0.0400 & 0.0400 & 0.0098 \\
\hline 0.2 & -0.6 & -0.2000 & -0.2000 & -0.0108 & 0.0400 & 0.0400 & 0.0102 \\
\hline 0.2 & -0.4 & -0.2000 & -0.2000 & -0.0060 & 0.0400 & 0.0400 & 0.0094 \\
\hline 0.2 & -0.2 & -0.1870 & -0.1799 & -0.0069 & 0.0373 & 0.0354 & 0.0096 \\
\hline 0.2 & 0 & -0.0432 & -0.0661 & -0.0089 & 0.0154 & 0.0200 & 0.0089 \\
\hline 0.2 & 0.2 & -0.0653 & -0.0655 & -0.0115 & 0.0439 & 0.0440 & 0.0085 \\
\hline 0.2 & 0.4 & -0.1756 & -0.1756 & -0.0052 & 0.0464 & 0.0464 & 0.0089 \\
\hline 0.2 & 0.6 & -0.2000 & -0.2000 & -0.0070 & 0.0400 & 0.0400 & 0.0085 \\
\hline 0.2 & 0.8 & -0.2000 & -0.2000 & -0.0120 & 0.0400 & 0.0400 & 0.0079 \\
\hline 0.4 & -0.8 & -0.4000 & -0.4000 & -0.0106 & 0.1600 & 0.1600 & 0.0068 \\
\hline 0.4 & -0.6 & -0.4000 & -0.4000 & -0.0107 & 0.1600 & 0.1600 & 0.0071 \\
\hline 0.4 & -0.4 & -0.3591 & -0.3584 & -0.0103 & 0.1381 & 0.1377 & 0.0075 \\
\hline 0.4 & -0.2 & -0.1519 & -0.1461 & -0.0052 & 0.0339 & 0.0301 & 0.0079 \\
\hline 0.4 & 0 & -0.0074 & -0.0085 & -0.0055 & 0.0062 & 0.0069 & 0.0077 \\
\hline 0.4 & 0.2 & 0.0895 & 0.0895 & -0.0066 & 0.0488 & 0.0488 & 0.0073 \\
\hline 0.4 & 0.4 & -0.0560 & -0.0560 & -0.0041 & 0.1399 & 0.1399 & 0.0070 \\
\hline 0.4 & 0.6 & -0.3321 & -0.3321 & -0.0030 & 0.1649 & 0.1649 & 0.0071 \\
\hline 0.4 & 0.8 & -0.4000 & -0.4000 & -0.0080 & 0.1600 & 0.1600 & 0.0063 \\
\hline 0.6 & -0.8 & -0.5968 & -0.5968 & -0.0074 & 0.3575 & 0.3575 & 0.0039 \\
\hline 0.6 & -0.6 & -0.4416 & -0.4416 & -0.0109 & 0.2262 & 0.2262 & 0.0049 \\
\hline 0.6 & -0.4 & -0.2258 & -0.2254 & -0.0122 & 0.0588 & 0.0583 & 0.0051 \\
\hline 0.6 & -0.2 & -0.1201 & -0.1201 & -0.0112 & 0.0186 & 0.0186 & 0.0060 \\
\hline 0.6 & 0 & -0.0047 & -0.0047 & -0.0100 & 0.0030 & 0.0030 & 0.0060 \\
\hline 0.6 & 0.2 & 0.1088 & 0.1088 & -0.0151 & 0.0200 & 0.0200 & 0.0067 \\
\hline 0.6 & 0.4 & 0.1602 & 0.1602 & -0.0113 & 0.0801 & 0.0801 & 0.0064 \\
\hline 0.6 & 0.6 & -0.1102 & -0.1102 & -0.0050 & 0.2225 & 0.2225 & 0.0058 \\
\hline 0.6 & 0.8 & -0.5630 & -0.5630 & -0.0056 & 0.3516 & 0.3516 & 0.0056 \\
\hline 0.8 & -0.8 & -0.3412 & -0.3412 & -0.0045 & 0.1281 & 0.1281 & 0.0017 \\
\hline 0.8 & -0.6 & -0.2418 & -0.2418 & -0.0068 & 0.0629 & 0.0629 & 0.0018 \\
\hline 0.8 & -0.4 & -0.1570 & -0.1570 & -0.0067 & 0.0277 & 0.0277 & 0.0021 \\
\hline 0.8 & -0.2 & -0.0794 & -0.0794 & -0.0100 & 0.0082 & 0.0082 & 0.0025 \\
\hline 0.8 & 0 & -0.0032 & -0.0032 & -0.0098 & 0.0012 & 0.0012 & 0.0028 \\
\hline 0.8 & 0.2 & 0.0636 & 0.0636 & -0.0107 & 0.0047 & 0.0047 & 0.0034 \\
\hline 0.8 & 0.4 & 0.1190 & 0.1190 & -0.0113 & 0.0170 & 0.0170 & 0.0034 \\
\hline 0.8 & 0.6 & 0.0474 & 0.0474 & -0.0138 & 0.1021 & 0.1021 & 0.0045 \\
\hline 0.8 & 0.8 & -0.4702 & -0.4702 & -0.0044 & 0.4390 & 0.4390 & 0.0040 \\
\hline ave & & 0.2583 & 0.2587 & 0.0079 & 0.1417 & 0.1417 & 0.0087 \\
\hline
\end{tabular}


Table 2: Bias and MSE of $\widehat{\lambda}_{P T 1}, \widehat{\lambda}_{P T 2}$, and $\widehat{\lambda}_{M L}$.

\begin{tabular}{|c|c|c|c|c|c|c|c|}
\hline \multirow[b]{2}{*}{$\rho$} & \multirow[b]{2}{*}{$\lambda$} & \multicolumn{3}{|c|}{ BIAS } & & MSE & \\
\hline & & $\widehat{\lambda}_{P T 1}$ & $\widehat{\lambda}_{P T 2}$ & $\widehat{\lambda}_{M L}$ & $\widehat{\lambda}_{P T 1}$ & $\widehat{\lambda}_{P T 2}$ & $\widehat{\lambda}_{M L}$ \\
\hline-0.8 & -0.8 & -0.2884 & -0.2884 & -0.0035 & 0.0860 & 0.0860 & 0.0047 \\
\hline-0.8 & -0.6 & -0.2893 & -0.2893 & 0.0003 & 0.0897 & 0.0897 & 0.0043 \\
\hline-0.8 & -0.4 & -0.1936 & -0.1936 & -0.0022 & 0.0986 & 0.0986 & 0.0035 \\
\hline-0.8 & -0.2 & 0.1264 & 0.1264 & -0.0042 & 0.0490 & 0.0490 & 0.0031 \\
\hline-0.8 & 0 & 0.0000 & 0.0000 & -0.0044 & 0.0000 & 0.0000 & 0.0024 \\
\hline-0.8 & 0.2 & -0.1998 & -0.1998 & -0.0014 & 0.0399 & 0.0399 & 0.0016 \\
\hline-0.8 & 0.4 & -0.1543 & -0.1543 & -0.0019 & 0.0251 & 0.0251 & 0.0010 \\
\hline-0.8 & 0.6 & -0.0995 & -0.0995 & -0.0012 & 0.0105 & 0.0105 & 0.0005 \\
\hline-0.8 & 0.8 & -0.0442 & -0.0442 & -0.0007 & 0.0022 & 0.0022 & 0.0001 \\
\hline-0.6 & -0.8 & -0.2116 & -0.2116 & 0.0010 & 0.0474 & 0.0474 & 0.0046 \\
\hline-0.6 & -0.6 & -0.2168 & -0.2168 & -0.0012 & 0.0497 & 0.0497 & 0.0037 \\
\hline-0.6 & -0.4 & -0.1884 & -0.1884 & -0.0016 & 0.0512 & 0.0512 & 0.0036 \\
\hline-0.6 & -0.2 & 0.0483 & 0.0483 & -0.0026 & 0.0449 & 0.0449 & 0.0032 \\
\hline-0.6 & 0 & -0.0003 & -0.0003 & -0.0003 & 0.0001 & 0.0001 & 0.0023 \\
\hline-0.6 & 0.2 & -0.1852 & -0.1847 & -0.0023 & 0.0358 & 0.0356 & 0.0017 \\
\hline-0.6 & 0.4 & -0.1143 & -0.1143 & -0.0019 & 0.0142 & 0.0142 & 0.0010 \\
\hline-0.6 & 0.6 & -0.0743 & -0.0743 & -0.0007 & 0.0061 & 0.0061 & 0.0005 \\
\hline-0.6 & 0.8 & -0.0329 & -0.0329 & -0.0002 & 0.0013 & 0.0013 & 0.0001 \\
\hline-0.4 & -0.8 & -0.1437 & -0.1437 & 0.0016 & 0.0233 & 0.0233 & 0.0044 \\
\hline-0.4 & -0.6 & -0.1422 & -0.1422 & 0.0053 & 0.0233 & 0.0233 & 0.0046 \\
\hline-0.4 & -0.4 & -0.1343 & -0.1343 & 0.0019 & 0.0232 & 0.0232 & 0.0036 \\
\hline-0.4 & -0.2 & -0.0172 & -0.0172 & -0.0003 & 0.0302 & 0.0302 & 0.0034 \\
\hline-0.4 & 0 & -0.0050 & -0.0035 & 0.0000 & 0.0010 & 0.0008 & 0.0028 \\
\hline-0.4 & 0.2 & -0.1238 & -0.1184 & -0.0045 & 0.0191 & 0.0174 & 0.0020 \\
\hline-0.4 & 0.4 & -0.0756 & -0.0756 & 0.0009 & 0.0069 & 0.0069 & 0.0012 \\
\hline-0.4 & 0.6 & -0.0537 & -0.0537 & -0.0029 & 0.0035 & 0.0035 & 0.0006 \\
\hline-0.4 & 0.8 & -0.0242 & -0.0242 & -0.0013 & 0.0008 & 0.0008 & 0.0002 \\
\hline-0.2 & -0.8 & -0.0737 & -0.0737 & 0.0014 & 0.0085 & 0.0085 & 0.0047 \\
\hline-0.2 & -0.6 & -0.0724 & -0.0724 & 0.0059 & 0.0079 & 0.0079 & 0.0043 \\
\hline-0.2 & -0.4 & -0.0718 & -0.0718 & 0.0029 & 0.0080 & 0.0080 & 0.0044 \\
\hline-0.2 & -0.2 & -0.0452 & -0.0400 & -0.0041 & 0.0123 & 0.0134 & 0.0036 \\
\hline-0.2 & 0 & -0.0104 & -0.0023 & -0.0013 & 0.0014 & 0.0004 & 0.0028 \\
\hline-0.2 & 0.2 & -0.0594 & -0.0571 & -0.0033 & 0.0061 & 0.0054 & 0.0021 \\
\hline-0.2 & 0.4 & -0.0420 & -0.0420 & -0.0022 & 0.0030 & 0.0030 & 0.0015 \\
\hline-0.2 & 0.6 & -0.0292 & -0.0292 & -0.0026 & 0.0015 & 0.0015 & 0.0007 \\
\hline-0.2 & 0.8 & -0.0141 & -0.0141 & -0.0020 & 0.0004 & 0.0004 & 0.0002 \\
\hline 0 & -0.8 & -0.0033 & -0.0033 & 0.0006 & 0.0031 & 0.0031 & 0.0047 \\
\hline 0 & -0.6 & 0.0046 & 0.0046 & 0.0065 & 0.0031 & 0.0031 & 0.0047 \\
\hline 0 & -0.4 & -0.0042 & -0.0042 & -0.0024 & 0.0030 & 0.0030 & 0.0045 \\
\hline 0 & -0.2 & 0.0008 & 0.0094 & -0.0025 & 0.0045 & 0.0067 & 0.0044 \\
\hline 0 & 0 & 0.0000 & 0.0001 & 0.0021 & 0.0006 & 0.0005 & 0.0034 \\
\hline 0 & 0.2 & -0.0077 & -0.0102 & -0.0027 & 0.0028 & 0.0035 & 0.0025 \\
\hline 0 & 0.4 & -0.0014 & -0.0014 & -0.0024 & 0.0012 & 0.0012 & 0.0017 \\
\hline 0 & 0.6 & -0.0022 & -0.0022 & -0.0024 & 0.0007 & 0.0007 & 0.0010 \\
\hline 0 & 0.8 & -0.0012 & -0.0012 & -0.0013 & 0.0002 & 0.0002 & 0.0003 \\
\hline 0.2 & -0.8 & 0.0814 & 0.0814 & 0.0023 & 0.0101 & 0.0101 & 0.0052 \\
\hline 0.2 & -0.6 & 0.0860 & 0.0860 & 0.0025 & 0.0110 & 0.0110 & 0.0054 \\
\hline 0.2 & -0.4 & 0.0856 & 0.0856 & 0.0013 & 0.0104 & 0.0104 & 0.0050 \\
\hline 0.2 & -0.2 & 0.1079 & 0.0943 & -0.0010 & 0.0181 & 0.0138 & 0.0048 \\
\hline 0.2 & 0 & 0.0099 & 0.0048 & -0.0025 & 0.0013 & 0.0008 & 0.0041 \\
\hline 0.2 & 0.2 & -0.0202 & -0.0202 & -0.0027 & 0.0168 & 0.0168 & 0.0031 \\
\hline 0.2 & 0.4 & 0.0296 & 0.0296 & -0.0037 & 0.0091 & 0.0091 & 0.0022 \\
\hline 0.2 & 0.6 & 0.0309 & 0.0309 & -0.0030 & 0.0017 & 0.0017 & 0.0012 \\
\hline 0.2 & 0.8 & 0.0133 & 0.0133 & -0.0028 & 0.0004 & 0.0004 & 0.0004 \\
\hline 0.4 & -0.8 & 0.1954 & 0.1954 & 0.0045 & 0.0422 & 0.0422 & 0.0053 \\
\hline 0.4 & -0.6 & 0.1980 & 0.1980 & 0.0015 & 0.0431 & 0.0431 & 0.0061 \\
\hline 0.4 & -0.4 & 0.2191 & 0.2183 & 0.0040 & 0.0572 & 0.0566 & 0.0062 \\
\hline 0.4 & -0.2 & 0.1952 & 0.1941 & -0.0034 & 0.0388 & 0.0384 & 0.0063 \\
\hline 0.4 & 0 & 0.0023 & 0.0022 & -0.0044 & 0.0005 & 0.0005 & 0.0056 \\
\hline 0.4 & 0.2 & -0.1510 & -0.1510 & -0.0042 & 0.0380 & 0.0380 & 0.0045 \\
\hline 0.4 & 0.4 & -0.1234 & -0.1234 & -0.0051 & 0.0824 & 0.0824 & 0.0032 \\
\hline 0.4 & 0.6 & 0.0249 & 0.0249 & -0.0053 & 0.0344 & 0.0344 & 0.0019 \\
\hline 0.4 & 0.8 & 0.0384 & 0.0384 & -0.0024 & 0.0017 & 0.0017 & 0.0006 \\
\hline 0.6 & -0.8 & 0.3715 & 0.3715 & 0.0061 & 0.1441 & 0.1441 & 0.0055 \\
\hline 0.6 & -0.6 & 0.4539 & 0.4539 & 0.0068 & 0.2260 & 0.2260 & 0.0071 \\
\hline 0.6 & -0.4 & 0.3971 & 0.3971 & 0.0060 & 0.1583 & 0.1583 & 0.0072 \\
\hline 0.6 & -0.2 & 0.2000 & 0.2000 & 0.0015 & 0.0400 & 0.0400 & 0.0081 \\
\hline 0.6 & 0 & 0.0000 & 0.0000 & -0.0005 & 0.0000 & 0.0000 & 0.0074 \\
\hline 0.6 & 0.2 & -0.1939 & -0.1939 & 0.0039 & 0.0404 & 0.0404 & 0.0070 \\
\hline 0.6 & 0.4 & -0.3480 & -0.3480 & -0.0018 & 0.1503 & 0.1503 & 0.0056 \\
\hline 0.6 & 0.6 & -0.2496 & -0.2496 & -0.0044 & 0.2027 & 0.2027 & 0.0036 \\
\hline 0.6 & 0.8 & 0.0426 & 0.0426 & -0.0073 & 0.0301 & 0.0301 & 0.0015 \\
\hline 0.8 & -0.8 & 0.7923 & 0.7923 & 0.0041 & 0.6299 & 0.6299 & 0.0059 \\
\hline 0.8 & -0.6 & 0.6000 & 0.6000 & 0.0004 & 0.3600 & 0.3600 & 0.0063 \\
\hline 0.8 & -0.4 & 0.4000 & 0.4000 & 0.0031 & 0.1600 & 0.1600 & 0.0079 \\
\hline 0.8 & -0.2 & 0.2000 & 0.2000 & 0.0078 & 0.0400 & 0.0400 & 0.0088 \\
\hline 0.8 & 0 & 0.0000 & 0.0000 & 0.0077 & 0.0000 & 0.0000 & 0.0087 \\
\hline 0.8 & 0.2 & -0.2000 & -0.2000 & 0.0000 & 0.0400 & 0.0400 & 0.0090 \\
\hline 0.8 & 0.4 & -0.3977 & -0.3977 & -0.0007 & 0.1599 & 0.1599 & 0.0084 \\
\hline 0.8 & 0.6 & -0.4962 & -0.4962 & -0.0020 & 0.3238 & 0.3238 & 0.0063 \\
\hline 0.8 & 0.8 & -0.1734 & -0.1734 & -0.0113 & 0.2262 & 0.2262 & 0.0037 \\
\hline ave & & 0.1328 & 0.1324 & 0.0029 & 0.0519 & 0.0518 & 0.0038 \\
\hline
\end{tabular}


Table 3: Bias and MSE of $\widehat{\beta}_{1, P T 1}, \widehat{\beta}_{1, P T 2}$, and $\widehat{\beta}_{1, M L}$.

\begin{tabular}{|c|c|c|c|c|c|c|c|}
\hline \multirow[b]{2}{*}{$\rho$} & \multirow[b]{2}{*}{$\lambda$} & \multicolumn{3}{|c|}{ BIAS } & & MSE & \\
\hline & & $\widehat{\boldsymbol{\beta}}_{1, P T 1}$ & $\widehat{\beta}_{1, P T 2}$ & $\widehat{\beta}_{1, M L}$ & $\widehat{\beta}_{1, P T 1}$ & $\widehat{\boldsymbol{\beta}}_{1, P T 2}$ & $\widehat{\beta}_{1, M L}$ \\
\hline-0.8 & -0.8 & 0.0075 & 0.0075 & 0.0003 & 0.0020 & 0.0020 & 0.0018 \\
\hline-0.8 & -0.6 & 0.0174 & 0.0174 & -0.0008 & 0.0025 & 0.0025 & 0.0018 \\
\hline-0.8 & -0.4 & 0.0020 & 0.0020 & 0.0001 & 0.0082 & 0.0082 & 0.0019 \\
\hline-0.8 & -0.2 & -0.0801 & -0.0801 & 0.0020 & 0.0115 & 0.0115 & 0.0020 \\
\hline-0.8 & 0 & -0.0013 & -0.0013 & 0.0006 & 0.0014 & 0.0014 & 0.0020 \\
\hline-0.8 & 0.2 & 0.1207 & 0.1207 & 0.0007 & 0.0165 & 0.0165 & 0.0022 \\
\hline-0.8 & 0.4 & 0.0620 & 0.0620 & 0.0004 & 0.0066 & 0.0066 & 0.0022 \\
\hline-0.8 & 0.6 & 0.0615 & 0.0615 & 0.0019 & 0.0066 & 0.0066 & 0.0023 \\
\hline-0.8 & 0.8 & 0.0489 & 0.0489 & -0.0021 & 0.0055 & 0.0055 & 0.0024 \\
\hline-0.6 & -0.8 & 0.0048 & 0.0048 & -0.0016 & 0.0020 & 0.0020 & 0.0019 \\
\hline-0.6 & -0.6 & 0.0144 & 0.0144 & 0.0001 & 0.0022 & 0.0022 & 0.0018 \\
\hline-0.6 & -0.4 & 0.0149 & 0.0149 & -0.0002 & 0.0035 & 0.0035 & 0.0018 \\
\hline-0.6 & -0.2 & -0.0455 & -0.0455 & -0.0003 & 0.0084 & 0.0084 & 0.0021 \\
\hline-0.6 & 0 & 0.0018 & 0.0018 & 0.0012 & 0.0015 & 0.0015 & 0.0020 \\
\hline-0.6 & 0.2 & 0.0877 & 0.0878 & 0.0000 & 0.0105 & 0.0106 & 0.0022 \\
\hline-0.6 & 0.4 & 0.0426 & 0.0426 & -0.0013 & 0.0042 & 0.0042 & 0.0022 \\
\hline-0.6 & 0.6 & 0.0462 & 0.0462 & 0.0004 & 0.0047 & 0.0047 & 0.0023 \\
\hline-0.6 & 0.8 & 0.0395 & 0.0395 & 0.0004 & 0.0044 & 0.0044 & 0.0024 \\
\hline-0.4 & -0.8 & 0.0024 & 0.0024 & -0.0016 & 0.0019 & 0.0019 & 0.0018 \\
\hline-0.4 & -0.6 & 0.0079 & 0.0079 & -0.0025 & 0.0019 & 0.0019 & 0.0019 \\
\hline-0.4 & -0.4 & 0.0113 & 0.0113 & -0.0023 & 0.0024 & 0.0024 & 0.0020 \\
\hline-0.4 & -0.2 & -0.0128 & -0.0128 & 0.0013 & 0.0048 & 0.0048 & 0.0020 \\
\hline-0.4 & 0 & -0.0023 & -0.0021 & -0.0046 & 0.0017 & 0.0017 & 0.0021 \\
\hline-0.4 & 0.2 & 0.0409 & 0.0405 & 0.0017 & 0.0046 & 0.0046 & 0.0023 \\
\hline-0.4 & 0.4 & 0.0277 & 0.0277 & -0.0025 & 0.0030 & 0.0030 & 0.0021 \\
\hline-0.4 & 0.6 & 0.0334 & 0.0334 & 0.0026 & 0.0035 & 0.0035 & 0.0023 \\
\hline-0.4 & 0.8 & 0.0286 & 0.0286 & 0.0025 & 0.0034 & 0.0034 & 0.0024 \\
\hline-0.2 & -0.8 & 0.0012 & 0.0012 & -0.0010 & 0.0019 & 0.0019 & 0.0018 \\
\hline-0.2 & -0.6 & 0.0016 & 0.0016 & -0.0036 & 0.0020 & 0.0020 & 0.0020 \\
\hline-0.2 & -0.4 & 0.0068 & 0.0068 & -0.0010 & 0.0019 & 0.0019 & 0.0019 \\
\hline-0.2 & -0.2 & 0.0032 & 0.0026 & 0.0006 & 0.0026 & 0.0026 & 0.0020 \\
\hline-0.2 & 0 & 0.0017 & 0.0011 & -0.0014 & 0.0020 & 0.0020 & 0.0022 \\
\hline-0.2 & 0.2 & 0.0142 & 0.0137 & -0.0014 & 0.0024 & 0.0024 & 0.0022 \\
\hline-0.2 & 0.4 & 0.0171 & 0.0171 & 0.0007 & 0.0024 & 0.0024 & 0.0022 \\
\hline-0.2 & 0.6 & 0.0201 & 0.0201 & 0.0036 & 0.0028 & 0.0028 & 0.0024 \\
\hline-0.2 & 0.8 & 0.0167 & 0.0167 & 0.0025 & 0.0025 & 0.0025 & 0.0023 \\
\hline 0 & -0.8 & -0.0005 & -0.0005 & -0.0007 & 0.0022 & 0.0022 & 0.0022 \\
\hline 0 & -0.6 & 0.0002 & 0.0002 & -0.0003 & 0.0020 & 0.0020 & 0.0020 \\
\hline 0 & -0.4 & 0.0012 & 0.0012 & 0.0007 & 0.0017 & 0.0017 & 0.0018 \\
\hline 0 & -0.2 & -0.0019 & -0.0032 & -0.0012 & 0.0021 & 0.0022 & 0.0021 \\
\hline 0 & 0 & -0.0017 & -0.0014 & -0.0031 & 0.0022 & 0.0022 & 0.0023 \\
\hline 0 & 0.2 & 0.0006 & 0.0012 & -0.0005 & 0.0022 & 0.0022 & 0.0022 \\
\hline 0 & 0.4 & 0.0009 & 0.0009 & 0.0009 & 0.0024 & 0.0024 & 0.0025 \\
\hline 0 & 0.6 & 0.0008 & 0.0008 & 0.0006 & 0.0023 & 0.0023 & 0.0024 \\
\hline 0 & 0.8 & -0.0008 & -0.0008 & -0.0009 & 0.0025 & 0.0025 & 0.0025 \\
\hline 0.2 & -0.8 & -0.0030 & -0.0030 & -0.0012 & 0.0022 & 0.0022 & 0.0021 \\
\hline 0.2 & -0.6 & -0.0072 & -0.0072 & -0.0016 & 0.0023 & 0.0023 & 0.0022 \\
\hline 0.2 & -0.4 & -0.0092 & -0.0092 & -0.0007 & 0.0021 & 0.0021 & 0.0020 \\
\hline 0.2 & -0.2 & -0.0140 & -0.0113 & 0.0000 & 0.0025 & 0.0023 & 0.0022 \\
\hline 0.2 & 0 & -0.0034 & -0.0013 & -0.0022 & 0.0022 & 0.0022 & 0.0022 \\
\hline 0.2 & 0.2 & -0.0093 & -0.0093 & -0.0008 & 0.0025 & 0.0025 & 0.0023 \\
\hline 0.2 & 0.4 & -0.0182 & -0.0182 & 0.0004 & 0.0025 & 0.0025 & 0.0023 \\
\hline 0.2 & 0.6 & -0.0193 & -0.0193 & 0.0009 & 0.0027 & 0.0027 & 0.0023 \\
\hline 0.2 & 0.8 & -0.0177 & -0.0177 & 0.0008 & 0.0025 & 0.0025 & 0.0022 \\
\hline 0.4 & -0.8 & -0.0074 & -0.0074 & -0.0010 & 0.0027 & 0.0027 & 0.0024 \\
\hline 0.4 & -0.6 & -0.0119 & -0.0119 & 0.0002 & 0.0026 & 0.0026 & 0.0024 \\
\hline 0.4 & -0.4 & -0.0117 & -0.0115 & 0.0031 & 0.0026 & 0.0026 & 0.0022 \\
\hline 0.4 & -0.2 & -0.0039 & -0.0033 & -0.0015 & 0.0024 & 0.0024 & 0.0023 \\
\hline 0.4 & 0 & -0.0004 & -0.0004 & -0.0013 & 0.0023 & 0.0024 & 0.0023 \\
\hline 0.4 & 0.2 & -0.0132 & -0.0132 & 0.0005 & 0.0023 & 0.0023 & 0.0021 \\
\hline 0.4 & 0.4 & -0.0407 & -0.0407 & -0.0016 & 0.0039 & 0.0039 & 0.0022 \\
\hline 0.4 & 0.6 & -0.0482 & -0.0482 & 0.0012 & 0.0047 & 0.0047 & 0.0023 \\
\hline 0.4 & 0.8 & -0.0493 & -0.0493 & -0.0022 & 0.0050 & 0.0050 & 0.0023 \\
\hline 0.6 & -0.8 & -0.0097 & -0.0097 & 0.0008 & 0.0036 & 0.0036 & 0.0027 \\
\hline 0.6 & -0.6 & 0.0121 & 0.0121 & -0.0010 & 0.0047 & 0.0047 & 0.0026 \\
\hline 0.6 & -0.4 & 0.0310 & 0.0311 & 0.0014 & 0.0038 & 0.0038 & 0.0026 \\
\hline 0.6 & -0.2 & 0.0184 & 0.0184 & -0.0003 & 0.0028 & 0.0028 & 0.0025 \\
\hline 0.6 & 0 & -0.0002 & -0.0002 & -0.0011 & 0.0023 & 0.0023 & 0.0025 \\
\hline 0.6 & 0.2 & -0.0235 & -0.0235 & 0.0017 & 0.0029 & 0.0029 & 0.0024 \\
\hline 0.6 & 0.4 & -0.0540 & -0.0540 & 0.0013 & 0.0052 & 0.0052 & 0.0025 \\
\hline 0.6 & 0.6 & -0.0788 & -0.0788 & 0.0012 & 0.0084 & 0.0084 & 0.0022 \\
\hline 0.6 & 0.8 & -0.0918 & -0.0918 & -0.0002 & 0.0112 & 0.0112 & 0.0024 \\
\hline 0.8 & -0.8 & 0.1401 & 0.1401 & 0.0016 & 0.0240 & 0.0240 & 0.0028 \\
\hline 0.8 & -0.6 & 0.1084 & 0.1084 & 0.0011 & 0.0148 & 0.0148 & 0.0027 \\
\hline 0.8 & -0.4 & 0.0695 & 0.0695 & -0.0019 & 0.0077 & 0.0077 & 0.0028 \\
\hline 0.8 & -0.2 & 0.0364 & 0.0364 & 0.0020 & 0.0039 & 0.0039 & 0.0027 \\
\hline 0.8 & 0 & -0.0006 & -0.0006 & 0.0000 & 0.0024 & 0.0024 & 0.0027 \\
\hline 0.8 & 0.2 & -0.0355 & -0.0355 & -0.0018 & 0.0033 & 0.0033 & 0.0023 \\
\hline 0.8 & 0.4 & -0.0638 & -0.0638 & 0.0024 & 0.0061 & 0.0061 & 0.0025 \\
\hline 0.8 & 0.6 & -0.0959 & -0.0959 & -0.0002 & 0.0111 & 0.0111 & 0.0021 \\
\hline 0.8 & 0.8 & -0.1412 & -0.1412 & 0.0000 & 0.0230 & 0.0230 & 0.0022 \\
\hline ave & & 0.0279 & 0.0278 & 0.0013 & 0.0044 & 0.0044 & 0.0022 \\
\hline
\end{tabular}


Table 4: Bias and MSE of $\widehat{\beta}_{2, P T 1}, \widehat{\beta}_{2, P T 2}$, and $\widehat{\beta}_{2, M L}$.

\begin{tabular}{|c|c|c|c|c|c|c|c|}
\hline \multirow[b]{2}{*}{$\rho$} & \multirow[b]{2}{*}{$\lambda$} & \multicolumn{3}{|c|}{ BIAS } & & MSE & \\
\hline & & $\widehat{\boldsymbol{\beta}}_{2, P T 1}$ & $\widehat{\beta}_{2, P T 2}$ & $\widehat{\beta}_{2, M L}$ & $\widehat{\beta}_{2, P T 1}$ & $\widehat{\boldsymbol{\beta}}_{2, P T 2}$ & $\widehat{\beta}_{2, M L}$ \\
\hline-0.8 & -0.8 & -0.0196 & -0.0196 & 0.0011 & 0.0025 & 0.0025 & 0.0018 \\
\hline-0.8 & -0.6 & -0.0149 & -0.0149 & -0.0003 & 0.0026 & 0.0026 & 0.0020 \\
\hline-0.8 & -0.4 & -0.0210 & -0.0210 & -0.0003 & 0.0047 & 0.0047 & 0.0020 \\
\hline-0.8 & -0.2 & -0.0556 & -0.0556 & -0.0014 & 0.0056 & 0.0056 & 0.0019 \\
\hline-0.8 & 0 & -0.0015 & -0.0015 & -0.0005 & 0.0017 & 0.0017 & 0.0019 \\
\hline-0.8 & 0.2 & 0.0621 & 0.0621 & 0.0003 & 0.0058 & 0.0058 & 0.0020 \\
\hline-0.8 & 0.4 & 0.0187 & 0.0187 & -0.0007 & 0.0029 & 0.0029 & 0.0021 \\
\hline-0.8 & 0.6 & 0.0231 & 0.0231 & 0.0012 & 0.0035 & 0.0035 & 0.0023 \\
\hline-0.8 & 0.8 & 0.0225 & 0.0225 & 0.0039 & 0.0031 & 0.0031 & 0.0019 \\
\hline-0.6 & -0.8 & -0.0181 & -0.0181 & -0.0026 & 0.0024 & 0.0024 & 0.0019 \\
\hline-0.6 & -0.6 & -0.0103 & -0.0103 & -0.0002 & 0.0023 & 0.0023 & 0.0019 \\
\hline-0.6 & -0.4 & -0.0062 & -0.0062 & 0.0006 & 0.0026 & 0.0026 & 0.0020 \\
\hline-0.6 & -0.2 & -0.0296 & -0.0296 & 0.0022 & 0.0041 & 0.0041 & 0.0023 \\
\hline-0.6 & 0 & 0.0022 & 0.0022 & 0.0017 & 0.0019 & 0.0019 & 0.0021 \\
\hline-0.6 & 0.2 & 0.0410 & 0.0411 & 0.0019 & 0.0041 & 0.0041 & 0.0021 \\
\hline-0.6 & 0.4 & 0.0165 & 0.0165 & 0.0009 & 0.0027 & 0.0027 & 0.0021 \\
\hline-0.6 & 0.6 & 0.0157 & 0.0157 & -0.0002 & 0.0026 & 0.0026 & 0.0020 \\
\hline-0.6 & 0.8 & 0.0134 & 0.0134 & -0.0003 & 0.0027 & 0.0027 & 0.0021 \\
\hline-0.4 & -0.8 & -0.0101 & -0.0101 & 0.0000 & 0.0023 & 0.0023 & 0.0021 \\
\hline-0.4 & -0.6 & -0.0096 & -0.0096 & -0.0029 & 0.0020 & 0.0020 & 0.0018 \\
\hline-0.4 & -0.4 & -0.0031 & -0.0031 & 0.0001 & 0.0023 & 0.0023 & 0.0022 \\
\hline-0.4 & -0.2 & -0.0166 & -0.0166 & -0.0037 & 0.0028 & 0.0028 & 0.0020 \\
\hline-0.4 & 0 & -0.0014 & -0.0013 & -0.0026 & 0.0019 & 0.0019 & 0.0020 \\
\hline-0.4 & 0.2 & 0.0144 & 0.0145 & 0.0002 & 0.0024 & 0.0025 & 0.0020 \\
\hline-0.4 & 0.4 & 0.0095 & 0.0095 & -0.0015 & 0.0022 & 0.0022 & 0.0020 \\
\hline-0.4 & 0.6 & 0.0125 & 0.0125 & 0.0003 & 0.0024 & 0.0024 & 0.0020 \\
\hline-0.4 & 0.8 & 0.0100 & 0.0100 & -0.0003 & 0.0025 & 0.0025 & 0.0022 \\
\hline-0.2 & -0.8 & -0.0065 & -0.0065 & -0.0016 & 0.0021 & 0.0021 & 0.0020 \\
\hline-0.2 & -0.6 & -0.0057 & -0.0057 & -0.0026 & 0.0022 & 0.0022 & 0.0021 \\
\hline-0.2 & -0.4 & -0.0021 & -0.0021 & -0.0013 & 0.0021 & 0.0021 & 0.0021 \\
\hline-0.2 & -0.2 & -0.0012 & -0.0012 & 0.0008 & 0.0023 & 0.0023 & 0.0021 \\
\hline-0.2 & 0 & 0.0004 & 0.0008 & -0.0007 & 0.0020 & 0.0020 & 0.0020 \\
\hline-0.2 & 0.2 & 0.0064 & 0.0062 & 0.0013 & 0.0022 & 0.0022 & 0.0021 \\
\hline-0.2 & 0.4 & 0.0070 & 0.0070 & 0.0018 & 0.0022 & 0.0022 & 0.0021 \\
\hline-0.2 & 0.6 & 0.0055 & 0.0055 & -0.0005 & 0.0021 & 0.0021 & 0.0021 \\
\hline-0.2 & 0.8 & 0.0078 & 0.0078 & 0.0023 & 0.0023 & 0.0023 & 0.0022 \\
\hline 0 & -0.8 & -0.0006 & -0.0006 & -0.0007 & 0.0021 & 0.0021 & 0.0021 \\
\hline 0 & -0.6 & 0.0008 & 0.0008 & 0.0007 & 0.0021 & 0.0021 & 0.0021 \\
\hline 0 & -0.4 & -0.0002 & -0.0002 & -0.0003 & 0.0022 & 0.0022 & 0.0023 \\
\hline 0 & -0.2 & 0.0012 & 0.0011 & 0.0012 & 0.0021 & 0.0021 & 0.0021 \\
\hline 0 & 0 & 0.0009 & 0.0011 & 0.0003 & 0.0020 & 0.0020 & 0.0020 \\
\hline 0 & 0.2 & 0.0004 & 0.0006 & 0.0003 & 0.0022 & 0.0021 & 0.0022 \\
\hline 0 & 0.4 & 0.0012 & 0.0012 & 0.0012 & 0.0022 & 0.0022 & 0.0022 \\
\hline 0 & 0.6 & -0.0002 & -0.0002 & -0.0002 & 0.0022 & 0.0022 & 0.0022 \\
\hline 0 & 0.8 & 0.0011 & 0.0011 & 0.0012 & 0.0021 & 0.0021 & 0.0021 \\
\hline 0.2 & -0.8 & 0.0037 & 0.0037 & -0.0019 & 0.0022 & 0.0022 & 0.0022 \\
\hline 0.2 & -0.6 & 0.0022 & 0.0022 & -0.0022 & 0.0023 & 0.0023 & 0.0023 \\
\hline 0.2 & -0.4 & 0.0022 & 0.0022 & 0.0004 & 0.0023 & 0.0023 & 0.0023 \\
\hline 0.2 & -0.2 & -0.0005 & 0.0001 & 0.0001 & 0.0022 & 0.0022 & 0.0021 \\
\hline 0.2 & 0 & 0.0017 & 0.0025 & 0.0013 & 0.0022 & 0.0022 & 0.0022 \\
\hline 0.2 & 0.2 & -0.0034 & -0.0034 & 0.0025 & 0.0022 & 0.0022 & 0.0021 \\
\hline 0.2 & 0.4 & -0.0066 & -0.0066 & 0.0009 & 0.0022 & 0.0022 & 0.0021 \\
\hline 0.2 & 0.6 & -0.0083 & -0.0083 & -0.0005 & 0.0022 & 0.0022 & 0.0021 \\
\hline 0.2 & 0.8 & -0.0085 & -0.0085 & -0.0019 & 0.0023 & 0.0023 & 0.0022 \\
\hline 0.4 & -0.8 & 0.0142 & 0.0142 & 0.0004 & 0.0026 & 0.0026 & 0.0023 \\
\hline 0.4 & -0.6 & 0.0067 & 0.0067 & -0.0020 & 0.0026 & 0.0026 & 0.0024 \\
\hline 0.4 & -0.4 & 0.0100 & 0.0100 & 0.0014 & 0.0025 & 0.0025 & 0.0022 \\
\hline 0.4 & -0.2 & 0.0131 & 0.0134 & -0.0016 & 0.0024 & 0.0024 & 0.0022 \\
\hline 0.4 & 0 & -0.0001 & -0.0001 & -0.0011 & 0.0021 & 0.0021 & 0.0021 \\
\hline 0.4 & 0.2 & -0.0189 & -0.0189 & -0.0007 & 0.0026 & 0.0026 & 0.0023 \\
\hline 0.4 & 0.4 & -0.0315 & -0.0315 & -0.0028 & 0.0033 & 0.0033 & 0.0020 \\
\hline 0.4 & 0.6 & -0.0209 & -0.0209 & 0.0005 & 0.0030 & 0.0030 & 0.0022 \\
\hline 0.4 & 0.8 & -0.0159 & -0.0159 & 0.0007 & 0.0024 & 0.0024 & 0.0021 \\
\hline 0.6 & -0.8 & 0.0252 & 0.0252 & -0.0018 & 0.0037 & 0.0037 & 0.0025 \\
\hline 0.6 & -0.6 & 0.0486 & 0.0486 & 0.0010 & 0.0064 & 0.0064 & 0.0024 \\
\hline 0.6 & -0.4 & 0.0503 & 0.0503 & -0.0002 & 0.0051 & 0.0051 & 0.0024 \\
\hline 0.6 & -0.2 & 0.0272 & 0.0272 & 0.0004 & 0.0029 & 0.0029 & 0.0022 \\
\hline 0.6 & 0 & 0.0000 & 0.0000 & -0.0008 & 0.0021 & 0.0021 & 0.0023 \\
\hline 0.6 & 0.2 & -0.0269 & -0.0269 & 0.0011 & 0.0027 & 0.0027 & 0.0022 \\
\hline 0.6 & 0.4 & -0.0567 & -0.0567 & -0.0028 & 0.0052 & 0.0052 & 0.0020 \\
\hline 0.6 & 0.6 & -0.0569 & -0.0569 & 0.0016 & 0.0061 & 0.0061 & 0.0021 \\
\hline 0.6 & 0.8 & -0.0355 & -0.0355 & -0.0006 & 0.0040 & 0.0040 & 0.0022 \\
\hline 0.8 & -0.8 & 0.1630 & 0.1630 & 0.0025 & 0.0301 & 0.0301 & 0.0025 \\
\hline 0.8 & -0.6 & 0.1154 & 0.1154 & -0.0021 & 0.0160 & 0.0160 & 0.0025 \\
\hline 0.8 & -0.4 & 0.0770 & 0.0770 & 0.0016 & 0.0088 & 0.0088 & 0.0027 \\
\hline 0.8 & -0.2 & 0.0363 & 0.0363 & 0.0009 & 0.0035 & 0.0035 & 0.0024 \\
\hline 0.8 & 0 & -0.0030 & -0.0030 & -0.0021 & 0.0021 & 0.0021 & 0.0025 \\
\hline 0.8 & 0.2 & -0.0331 & -0.0331 & 0.0000 & 0.0030 & 0.0030 & 0.0022 \\
\hline 0.8 & 0.4 & -0.0627 & -0.0627 & 0.0011 & 0.0057 & 0.0057 & 0.0021 \\
\hline 0.8 & 0.6 & -0.0872 & -0.0872 & -0.0033 & 0.0097 & 0.0097 & 0.0021 \\
\hline 0.8 & 0.8 & -0.0702 & -0.0702 & 0.0011 & 0.0080 & 0.0080 & 0.0020 \\
\hline ave & & 0.0206 & 0.0207 & 0.0012 & 0.0035 & 0.0035 & 0.0021 \\
\hline
\end{tabular}


Table 5: Frequency of Selecting OLS, Spatial Error and Spatial Lag Model with Pretest Procedures

\begin{tabular}{|c|c|c|c|c|c|c|c|}
\hline \multirow[b]{2}{*}{$\rho$} & \multirow[b]{2}{*}{$\lambda$} & \multicolumn{3}{|c|}{ Prestest Estimator 1} & \multicolumn{3}{|c|}{ Prestest Estimator 2} \\
\hline & & $\overline{\mathrm{OLS}}$ & Error Model & Lag Model & $\overline{\mathrm{OLS}}$ & Error Model & Lag Model \\
\hline-0.8 & -0.8 & 0.000 & 0.000 & 1.000 & 0.000 & 0.000 & 1.000 \\
\hline-0.8 & -0.6 & 0.000 & 0.004 & 0.996 & 0.000 & 0.004 & 0.996 \\
\hline-0.8 & -0.4 & 0.000 & 0.143 & 0.857 & 0.000 & 0.143 & 0.857 \\
\hline-0.8 & -0.2 & 0.000 & 0.858 & 0.142 & 0.000 & 0.858 & 0.142 \\
\hline-0.8 & 0 & 0.000 & 1.000 & 0.000 & 0.000 & 1.000 & 0.000 \\
\hline-0.8 & 0.2 & 0.001 & 0.997 & 0.002 & 0.000 & 0.998 & 0.002 \\
\hline-0.8 & 0.4 & 0.000 & 0.001 & 0.999 & 0.000 & 0.001 & 0.999 \\
\hline-0.8 & 0.6 & 0.000 & 0.000 & 1.000 & 0.000 & 0.000 & 1.000 \\
\hline-0.8 & 0.8 & 0.000 & 0.000 & 1.000 & 0.000 & 0.000 & 1.000 \\
\hline-0.6 & -0.8 & 0.000 & 0.000 & 1.000 & 0.000 & 0.000 & 1.000 \\
\hline-0.6 & -0.6 & 0.000 & 0.000 & 1.000 & 0.000 & 0.000 & 1.000 \\
\hline-0.6 & -0.4 & 0.000 & 0.037 & 0.963 & 0.000 & 0.037 & 0.963 \\
\hline-0.6 & -0.2 & 0.000 & 0.645 & 0.355 & 0.000 & 0.645 & 0.355 \\
\hline-0.6 & 0 & 0.000 & 0.999 & 0.001 & 0.000 & 0.999 & 0.001 \\
\hline-0.6 & 0.2 & 0.022 & 0.846 & 0.132 & 0.000 & 0.861 & 0.139 \\
\hline-0.6 & 0.4 & 0.000 & 0.000 & 1.000 & 0.000 & 0.000 & 1.000 \\
\hline-0.6 & 0.6 & 0.000 & 0.000 & 1.000 & 0.000 & 0.000 & 1.000 \\
\hline-0.6 & 0.8 & 0.000 & 0.000 & 1.000 & 0.000 & 0.000 & 1.000 \\
\hline-0.4 & -0.8 & 0.000 & 0.000 & 1.000 & 0.000 & 0.000 & 1.000 \\
\hline-0.4 & -0.6 & 0.000 & 0.000 & 1.000 & 0.000 & 0.000 & 1.000 \\
\hline-0.4 & -0.4 & 0.000 & 0.009 & 0.991 & 0.000 & 0.009 & 0.991 \\
\hline-0.4 & -0.2 & 0.000 & 0.377 & 0.623 & 0.000 & 0.377 & 0.623 \\
\hline-0.4 & 0 & 0.003 & 0.971 & 0.026 & 0.047 & 0.937 & 0.016 \\
\hline-0.4 & 0.2 & 0.147 & 0.220 & 0.633 & 0.005 & 0.288 & 0.707 \\
\hline-0.4 & 0.4 & 0.000 & 0.000 & 1.000 & 0.000 & 0.000 & 1.000 \\
\hline-0.4 & 0.6 & 0.000 & 0.000 & 1.000 & 0.000 & 0.000 & 1.000 \\
\hline-0.4 & 0.8 & 0.000 & 0.000 & 1.000 & 0.000 & 0.000 & 1.000 \\
\hline-0.2 & -0.8 & 0.000 & 0.000 & 1.000 & 0.000 & 0.000 & 1.000 \\
\hline-0.2 & -0.6 & 0.000 & 0.000 & 1.000 & 0.000 & 0.000 & 1.000 \\
\hline-0.2 & -0.4 & 0.000 & 0.000 & 1.000 & 0.000 & 0.000 & 1.000 \\
\hline-0.2 & -0.2 & 0.000 & 0.120 & 0.880 & 0.041 & 0.107 & 0.852 \\
\hline-0.2 & 0 & 0.380 & 0.541 & 0.079 & 0.565 & 0.418 & 0.017 \\
\hline-0.2 & 0.2 & 0.065 & 0.003 & 0.932 & 0.025 & 0.011 & 0.964 \\
\hline-0.2 & 0.4 & 0.000 & 0.000 & 1.000 & 0.000 & 0.000 & 1.000 \\
\hline-0.2 & 0.6 & 0.000 & 0.000 & 1.000 & 0.000 & 0.000 & 1.000 \\
\hline-0.2 & 0.8 & 0.000 & 0.000 & 1.000 & 0.000 & 0.000 & 1.000 \\
\hline 0 & -0.8 & 0.000 & 0.000 & 1.000 & 0.000 & 0.000 & 1.000 \\
\hline 0 & -0.6 & 0.000 & 0.000 & 1.000 & 0.000 & 0.000 & 1.000 \\
\hline 0 & -0.4 & 0.000 & 0.000 & 1.000 & 0.000 & 0.000 & 1.000 \\
\hline 0 & -0.2 & 0.027 & 0.024 & 0.949 & 0.104 & 0.008 & 0.888 \\
\hline 0 & 0 & 0.901 & 0.050 & 0.049 & 0.912 & 0.049 & 0.039 \\
\hline 0 & 0.2 & 0.006 & 0.026 & 0.968 & 0.030 & 0.023 & 0.947 \\
\hline 0 & 0.4 & 0.000 & 0.000 & 1.000 & 0.000 & 0.000 & 1.000 \\
\hline 0 & 0.6 & 0.000 & 0.000 & 1.000 & 0.000 & 0.000 & 1.000 \\
\hline 0 & 0.8 & 0.000 & 0.000 & 1.000 & 0.000 & 0.000 & 1.000 \\
\hline 0.2 & -0.8 & 0.000 & 0.000 & 1.000 & 0.000 & 0.000 & 1.000 \\
\hline 0.2 & -0.6 & 0.000 & 0.000 & 1.000 & 0.000 & 0.000 & 1.000 \\
\hline 0.2 & -0.4 & 0.000 & 0.000 & 1.000 & 0.000 & 0.000 & 1.000 \\
\hline 0.2 & -0.2 & 0.334 & 0.069 & 0.597 & 0.117 & 0.125 & 0.758 \\
\hline 0.2 & 0 & 0.243 & 0.676 & 0.081 & 0.401 & 0.560 & 0.039 \\
\hline 0.2 & 0.2 & 0.000 & 0.320 & 0.680 & 0.001 & 0.319 & 0.680 \\
\hline 0.2 & 0.4 & 0.000 & 0.037 & 0.963 & 0.000 & 0.037 & 0.963 \\
\hline 0.2 & 0.6 & 0.000 & 0.000 & 1.000 & 0.000 & 0.000 & 1.000 \\
\hline 0.2 & 0.8 & 0.000 & 0.000 & 1.000 & 0.000 & 0.000 & 1.000 \\
\hline 0.4 & -0.8 & 0.000 & 0.000 & 1.000 & 0.000 & 0.000 & 1.000 \\
\hline 0.4 & -0.6 & 0.000 & 0.000 & 1.000 & 0.000 & 0.000 & 1.000 \\
\hline 0.4 & -0.4 & 0.015 & 0.160 & 0.825 & 0.001 & 0.165 & 0.834 \\
\hline 0.4 & -0.2 & 0.070 & 0.895 & 0.035 & 0.013 & 0.938 & 0.049 \\
\hline 0.4 & 0 & 0.001 & 0.988 & 0.011 & 0.007 & 0.983 & 0.010 \\
\hline 0.4 & 0.2 & 0.000 & 0.862 & 0.138 & 0.000 & 0.862 & 0.138 \\
\hline 0.4 & 0.4 & 0.000 & 0.465 & 0.535 & 0.000 & 0.465 & 0.535 \\
\hline 0.4 & 0.6 & 0.000 & 0.078 & 0.922 & 0.000 & 0.078 & 0.922 \\
\hline 0.4 & 0.8 & 0.000 & 0.000 & 1.000 & 0.000 & 0.000 & 1.000 \\
\hline 0.6 & -0.8 & 0.000 & 0.008 & 0.992 & 0.000 & 0.008 & 0.992 \\
\hline 0.6 & -0.6 & 0.000 & 0.459 & 0.541 & 0.000 & 0.459 & 0.541 \\
\hline 0.6 & -0.4 & 0.003 & 0.982 & 0.015 & 0.000 & 0.985 & 0.015 \\
\hline 0.6 & -0.2 & 0.000 & 1.000 & 0.000 & 0.000 & 1.000 & 0.000 \\
\hline 0.6 & 0 & 0.000 & 1.000 & 0.000 & 0.000 & 1.000 & 0.000 \\
\hline 0.6 & 0.2 & 0.000 & 0.987 & 0.013 & 0.000 & 0.987 & 0.013 \\
\hline 0.6 & 0.4 & 0.000 & 0.915 & 0.085 & 0.000 & 0.915 & 0.085 \\
\hline 0.6 & 0.6 & 0.000 & 0.533 & 0.467 & 0.000 & 0.533 & 0.467 \\
\hline 0.6 & 0.8 & 0.000 & 0.038 & 0.962 & 0.000 & 0.038 & 0.962 \\
\hline 0.8 & -0.8 & 0.000 & 0.972 & 0.028 & 0.000 & 0.972 & 0.028 \\
\hline 0.8 & -0.6 & 0.000 & 1.000 & 0.000 & 0.000 & 1.000 & 0.000 \\
\hline 0.8 & -0.4 & 0.000 & 1.000 & 0.000 & 0.000 & 1.000 & 0.000 \\
\hline 0.8 & -0.2 & 0.000 & 1.000 & 0.000 & 0.000 & 1.000 & 0.000 \\
\hline 0.8 & 0 & 0.000 & 1.000 & 0.000 & 0.000 & 1.000 & 0.000 \\
\hline 0.8 & 0.2 & 0.000 & 1.000 & 0.000 & 0.000 & 1.000 & 0.000 \\
\hline 0.8 & 0.4 & 0.000 & 0.997 & 0.003 & 0.000 & 0.997 & 0.003 \\
\hline 0.8 & 0.6 & 0.000 & 0.878 & 0.122 & 0.000 & 0.878 & 0.122 \\
\hline 0.8 & 0.8 & 0.000 & 0.333 & 0.667 & 0.000 & 0.333 & 0.667 \\
\hline
\end{tabular}


Table 6: Size of Tests Associated with Pre-test and ML Estimators of the Hypothesis that a Respective Parameter is Equal to the True Parameter.

\begin{tabular}{|c|c|c|c|c|c|c|c|}
\hline$\rho$ & $\lambda$ & $\widehat{\beta}_{1, P T 1}$ & $\widehat{\beta}_{2, P T 1}$ & $\widehat{\boldsymbol{\beta}}_{1, P T 2}$ & $\widehat{\beta}_{2, P T 2}$ & $\widehat{\beta}_{1, M L}$ & $\widehat{\beta}_{2, M L}$ \\
\hline-0.8 & -0.8 & 0.0400 & 0.0550 & 0.0400 & 0.0550 & 0.0550 & 0.0430 \\
\hline-0.8 & -0.6 & 0.0560 & 0.0580 & 0.0560 & 0.0580 & 0.0540 & 0.0590 \\
\hline-0.8 & -0.4 & 0.2240 & 0.1770 & 0.2240 & 0.1770 & 0.0600 & 0.0680 \\
\hline-0.8 & -0.2 & 0.7870 & 0.3520 & 0.7870 & 0.3520 & 0.0480 & 0.0470 \\
\hline-0.8 & 0 & 0.0500 & 0.0580 & 0.0500 & 0.0580 & 0.0470 & 0.0540 \\
\hline-0.8 & 0.2 & 0.8270 & 0.2700 & 0.8270 & 0.2710 & 0.0540 & 0.0520 \\
\hline-0.8 & 0.4 & 0.2260 & 0.0640 & 0.2260 & 0.0640 & 0.0590 & 0.0600 \\
\hline-0.8 & 0.6 & 0.2210 & 0.1000 & 0.2210 & 0.1000 & 0.0560 & 0.0710 \\
\hline-0.8 & 0.8 & 0.1580 & 0.0770 & 0.1580 & 0.0770 & 0.0660 & 0.0600 \\
\hline-0.6 & -0.8 & 0.0530 & 0.0620 & 0.0530 & 0.0620 & 0.0650 & 0.0490 \\
\hline-0.6 & -0.6 & 0.0490 & 0.0470 & 0.0490 & 0.0470 & 0.0470 & 0.0510 \\
\hline-0.6 & -0.4 & 0.0940 & 0.0710 & 0.0940 & 0.0710 & 0.0520 & 0.0470 \\
\hline-0.6 & -0.2 & 0.5560 & 0.2110 & 0.5560 & 0.2110 & 0.0500 & 0.0780 \\
\hline-0.6 & 0 & 0.0430 & 0.0610 & 0.0430 & 0.0610 & 0.0450 & 0.0610 \\
\hline-0.6 & 0.2 & 0.5690 & 0.1670 & 0.5720 & 0.1670 & 0.0500 & 0.0620 \\
\hline-0.6 & 0.4 & 0.1260 & 0.0670 & 0.1260 & 0.0670 & 0.0490 & 0.0510 \\
\hline-0.6 & 0.6 & 0.1550 & 0.0620 & 0.1550 & 0.0620 & 0.0560 & 0.0400 \\
\hline-0.6 & 0.8 & 0.1380 & 0.0650 & 0.1380 & 0.0650 & 0.0540 & 0.0610 \\
\hline-0.4 & -0.8 & 0.0380 & 0.0570 & 0.0380 & 0.0570 & 0.0500 & 0.0500 \\
\hline-0.4 & -0.6 & 0.0500 & 0.0450 & 0.0500 & 0.0450 & 0.0590 & 0.0380 \\
\hline-0.4 & -0.4 & 0.0620 & 0.0620 & 0.0620 & 0.0620 & 0.0570 & 0.0680 \\
\hline-0.4 & -0.2 & 0.2630 & 0.0890 & 0.2630 & 0.0890 & 0.0440 & 0.0490 \\
\hline-0.4 & 0 & 0.0530 & 0.0440 & 0.0520 & 0.0440 & 0.0570 & 0.0420 \\
\hline-0.4 & 0.2 & 0.1960 & 0.0700 & 0.1950 & 0.0700 & 0.0540 & 0.0510 \\
\hline-0.4 & 0.4 & 0.0770 & 0.0490 & 0.0770 & 0.0490 & 0.0420 & 0.0560 \\
\hline-0.4 & 0.6 & 0.1030 & 0.0510 & 0.1030 & 0.0510 & 0.0530 & 0.0470 \\
\hline-0.4 & 0.8 & 0.0940 & 0.0620 & 0.0940 & 0.0620 & 0.0570 & 0.0570 \\
\hline-0.2 & -0.8 & 0.0410 & 0.0460 & 0.0410 & 0.0460 & 0.0440 & 0.0460 \\
\hline-0.2 & -0.6 & 0.0570 & 0.0560 & 0.0570 & 0.0560 & 0.0650 & 0.0670 \\
\hline-0.2 & -0.4 & 0.0390 & 0.0450 & 0.0390 & 0.0450 & 0.0450 & 0.0540 \\
\hline-0.2 & -0.2 & 0.0860 & 0.0610 & 0.0900 & 0.0590 & 0.0550 & 0.0510 \\
\hline-0.2 & 0 & 0.0590 & 0.0550 & 0.0470 & 0.0530 & 0.0550 & 0.0500 \\
\hline-0.2 & 0.2 & 0.0590 & 0.0520 & 0.0610 & 0.0510 & 0.0490 & 0.0510 \\
\hline-0.2 & 0.4 & 0.0550 & 0.0490 & 0.0550 & 0.0490 & 0.0500 & 0.0440 \\
\hline-0.2 & 0.6 & 0.0690 & 0.0430 & 0.0690 & 0.0430 & 0.0520 & 0.0480 \\
\hline-0.2 & 0.8 & 0.0570 & 0.0560 & 0.0570 & 0.0560 & 0.0440 & 0.0580 \\
\hline 0 & -0.8 & 0.0570 & 0.0480 & 0.0570 & 0.0480 & 0.0610 & 0.0490 \\
\hline 0 & -0.6 & 0.0500 & 0.0530 & 0.0500 & 0.0530 & 0.0540 & 0.0530 \\
\hline 0 & -0.4 & 0.0370 & 0.0550 & 0.0370 & 0.0550 & 0.0400 & 0.0580 \\
\hline 0 & -0.2 & 0.0650 & 0.0530 & 0.0660 & 0.0530 & 0.0530 & 0.0550 \\
\hline 0 & 0 & 0.0620 & 0.0440 & 0.0630 & 0.0450 & 0.0590 & 0.0430 \\
\hline 0 & 0.2 & 0.0510 & 0.0590 & 0.0530 & 0.0590 & 0.0520 & 0.0600 \\
\hline 0 & 0.4 & 0.0710 & 0.0520 & 0.0710 & 0.0520 & 0.0640 & 0.0510 \\
\hline 0 & 0.6 & 0.0640 & 0.0490 & 0.0640 & 0.0490 & 0.0560 & 0.0510 \\
\hline 0 & 0.8 & 0.0630 & 0.0510 & 0.0630 & 0.0510 & 0.0670 & 0.0490 \\
\hline 0.2 & -0.8 & 0.0510 & 0.0530 & 0.0510 & 0.0530 & 0.0430 & 0.0490 \\
\hline 0.2 & -0.6 & 0.0630 & 0.0600 & 0.0630 & 0.0600 & 0.0570 & 0.0580 \\
\hline 0.2 & -0.4 & 0.0470 & 0.0560 & 0.0470 & 0.0560 & 0.0440 & 0.0540 \\
\hline 0.2 & -0.2 & 0.0800 & 0.0560 & 0.0650 & 0.0550 & 0.0550 & 0.0490 \\
\hline 0.2 & 0 & 0.0560 & 0.0610 & 0.0600 & 0.0630 & 0.0520 & 0.0600 \\
\hline 0.2 & 0.2 & 0.0680 & 0.0430 & 0.0670 & 0.0430 & 0.0510 & 0.0490 \\
\hline 0.2 & 0.4 & 0.0740 & 0.0520 & 0.0740 & 0.0520 & 0.0450 & 0.0510 \\
\hline 0.2 & 0.6 & 0.0640 & 0.0520 & 0.0640 & 0.0520 & 0.0430 & 0.0520 \\
\hline 0.2 & 0.8 & 0.0550 & 0.0670 & 0.0550 & 0.0670 & 0.0300 & 0.0560 \\
\hline 0.4 & -0.8 & 0.0800 & 0.0700 & 0.0800 & 0.0700 & 0.0580 & 0.0540 \\
\hline 0.4 & -0.6 & 0.0790 & 0.0660 & 0.0790 & 0.0660 & 0.0540 & 0.0510 \\
\hline 0.4 & -0.4 & 0.0630 & 0.0790 & 0.0630 & 0.0790 & 0.0500 & 0.0490 \\
\hline 0.4 & -0.2 & 0.0620 & 0.0560 & 0.0600 & 0.0580 & 0.0520 & 0.0580 \\
\hline 0.4 & 0 & 0.0570 & 0.0540 & 0.0570 & 0.0540 & 0.0570 & 0.0550 \\
\hline 0.4 & 0.2 & 0.0480 & 0.0870 & 0.0480 & 0.0870 & 0.0450 & 0.0610 \\
\hline 0.4 & 0.4 & 0.1470 & 0.1140 & 0.1470 & 0.1140 & 0.0470 & 0.0410 \\
\hline 0.4 & 0.6 & 0.1710 & 0.1010 & 0.1710 & 0.1010 & 0.0490 & 0.0610 \\
\hline 0.4 & 0.8 & 0.1850 & 0.0580 & 0.1850 & 0.0580 & 0.0490 & 0.0540 \\
\hline 0.6 & -0.8 & 0.1080 & 0.0950 & 0.1080 & 0.0950 & 0.0630 & 0.0600 \\
\hline 0.6 & -0.6 & 0.1470 & 0.2330 & 0.1470 & 0.2330 & 0.0640 & 0.0630 \\
\hline 0.6 & -0.4 & 0.1040 & 0.1810 & 0.1040 & 0.1810 & 0.0580 & 0.0690 \\
\hline 0.6 & -0.2 & 0.0780 & 0.0950 & 0.0780 & 0.0950 & 0.0560 & 0.0440 \\
\hline 0.6 & 0 & 0.0520 & 0.0510 & 0.0520 & 0.0510 & 0.0570 & 0.0510 \\
\hline 0.6 & 0.2 & 0.0850 & 0.0930 & 0.0850 & 0.0930 & 0.0520 & 0.0510 \\
\hline 0.6 & 0.4 & 0.2140 & 0.2540 & 0.2140 & 0.2540 & 0.0700 & 0.0430 \\
\hline 0.6 & 0.6 & 0.3720 & 0.2900 & 0.3720 & 0.2900 & 0.0480 & 0.0590 \\
\hline 0.6 & 0.8 & 0.4230 & 0.1310 & 0.4230 & 0.1310 & 0.0530 & 0.0540 \\
\hline 0.8 & -0.8 & 0.7040 & 0.8260 & 0.7040 & 0.8260 & 0.0440 & 0.0450 \\
\hline 0.8 & -0.6 & 0.5650 & 0.6150 & 0.5650 & 0.6150 & 0.0500 & 0.0550 \\
\hline 0.8 & -0.4 & 0.2950 & 0.3780 & 0.2950 & 0.3780 & 0.0590 & 0.0640 \\
\hline 0.8 & -0.2 & 0.1240 & 0.1310 & 0.1240 & 0.1310 & 0.0530 & 0.0580 \\
\hline 0.8 & 0 & 0.0660 & 0.0670 & 0.0660 & 0.0670 & 0.0620 & 0.0660 \\
\hline 0.8 & 0.2 & 0.1150 & 0.1160 & 0.1150 & 0.1160 & 0.0410 & 0.0500 \\
\hline 0.8 & 0.4 & 0.2750 & 0.2690 & 0.2750 & 0.2690 & 0.0620 & 0.0510 \\
\hline 0.8 & 0.6 & 0.5030 & 0.5040 & 0.5030 & 0.5040 & 0.0370 & 0.0560 \\
\hline 0.8 & 0.8 & 0.7310 & 0.3330 & 0.7310 & 0.3330 & 0.0480 & 0.0530 \\
\hline \multicolumn{2}{|c|}{ average } & 0.1575 & 0.1151 & 0.1574 & 0.1151 & 0.0526 & 0.0539 \\
\hline
\end{tabular}

